\title{
Nature of crack-tip plastic zone in metallic glasses
}

\author{
Y. Chen, L.H. Dai* \\ State Key Laboratory of Nonlinear Mechanics, Institute of Mechanics, Chinese Academy of Sciences, Beijing 100190, People's Republic of \\ China
}

\section{A R T I C L E I N F O}

\section{Article history:}

Received 17 June 2015

Received in revised form 7 September 2015

Available online 23 October 2015

\section{Keywords:}

Metallic glass

A. Crack tip plasticity

A. Ductility

A. Stress intensity factor

C. Analytic functions

\begin{abstract}
A B S T R A C T
The fracture of metallic glasses(MGs) can be induced by shear banding in a ductile mode or by cavitation in a brittle way. Plastic zone in front of a crack tip, which is greatly involved with localized shear band, cavitation and the resultant fracture morphology, is a key clue to unveil the secrets of the intrinsic ductility and fracture. However, the characteristics of plastic zone, i.e., stress and strain distributions, size and shape, have not been clearly unraveled for MGs so far. In this paper, an analytical solution of the plastic zone for mode I crack under plane strain condition is derived through $J$-integral based on a slip line field analysis and shape approximation, by taking pressure-sensitivity, dilatancy, and structural evolution into account. Two length scales of the plastic zone, i.e. the maximum radius $R_{\max }$ and the radius along the crack line direction $R_{x}$, are revealed to control shear flow instability and cavitation, and therefore failure modes. According to shear transformation zone (STZ) based free volume evolution dynamics, the critical values of the mode I stress intensity factor and the plastic zone size at crack initiation are obtained. The effects of Poisson's ratio, pressure sensitivity, and dilatancy on the stress/strain distributions, and the size of plastic zone are elucidated. It is found that larger Poisson's ratio and smaller dilatancy lead to higher fracture toughness and 'slender' critical plastic zone, facilitating a good ductility. The internal correlations of the fracture pattern (i.e. dimple structure) with the plastic zone are established, where the size of the fracture pattern is quantitatively characterized by the critical length of plastic zone. To be further, a shape change of the critical plastic zone from 'slender' (apt to shear plastic flow) to 'chubby' (inclined to cavitation) is revealed with increasing dilatancy or decreasing Poisson's ratio, which might shed light on the underlying mechanism of ductile-to-brittle transition in MGs.
\end{abstract}

(c) 2015 Elsevier Ltd. All rights reserved.

\section{Introduction}

Compared with crystalline order, more materials in nature prefer to exist in a disordered state, e.g. noncrystalline/ amorphous solids and granular materials. Due to the absence of long-range structural order, plastic deformation in disordered solids is not accommodated by dislocation but through localized shear-driven rearrangements involving a small number of so-called plastic units (clusters of atoms/molecules/grains) (Spaepen, 1977; Argon, 1979; Falk and Langer, 1998). Some fundamental and unique mechanical traits are found in these materials (Rudnicki and Rice, 1975; Li and Pan, 1990a,b; Flores and Dauskardt, 2001; Schuh et al., 2007), such as the considerable pressure sensitivity in yielding, shear dilatancy during the

\footnotetext{
* Corresponding author. Tel.: +86 10 82543958; fax: +86 1082543977.

E-mail address: 1hdai@lnm.imech.ac.cn (L.H. Dai).
} 
deformation, and more complex failure behavior in contrast to that of ordered ones. The mysteries within the disordered materials arouse great interests of researchers but there still lots of problems remain elusive (Miracle, 2004; Falk and Langer, 2011; Biroli, 2014; Goodrich et al., 2014; Kamrin and Bouchbinder, 2014). As a new member of disordered materials, metallic glasses (MGs) behave as a double-blade sword for their extremely high strengths but poor room-temperature ductility. Their intrinsic inability to undergo finite plastic straining under tension greatly impedes MGs from wide structural usage. The underlying deformation and fracture physics, being one of the most fundamental problems of MGs, has attracted substantial research effort for the last decades (Spaepen, 1975; Argon and Salama, 1976; Ravichandran and Molinari, 2005; Schuh et al., 2007; Jiang et al., 2008a; Raghavan et al., 2009; Tandaiya et al., 2009; Xu et al., 2010; Chen et al., 2011; Jang et al., 2011; Greer et al., 2013; Tandaiya et al., 2013; Narayan et al., 2014; Narasimhan et al., 2015). Due to their special atomic structures, MGs may go through ductile failure via shear banding (Dai et al., 2005; Jiang et al., 2008b; Chen and Lin, 2010; Chen et al., 2013; Greer et al., 2013) or brittle fracture by cavitation (Jiang et al., 2008a; Murali et al., 2011a; Singh et al., 2013, 2014). At nanoscale, these inelastic deformation and fracture is accommodated by atomic clusters called as shear transformation zones (STZs) (Argon, 1979; Falk and Langer, 1998) or tension transformation zones (TTZs) (Jiang et al., 2008a; Huang et al., 2014a). The initiation and propagation of a shear band or a crack largely depend on the surrounding stress and deformation fields (Tandaiya et al., 2007, 2008; Huang et al., 2014b; Wu et al., 2015). As a critical region ahead of the crack tip, plastic zone may trigger or inhibit the shear banding or cracking and therefore determine the overall deformation and failure behavior, being a bridge between the micro-deformation and the macro-failure (Ashby and Greer, 2006).

From the viewpoint of phenomenological fracture mechanics, the ductile or brittle fracture through the initiation and growth of a crack depends on the surrounding stress and deformation fields near the tip. A number of important works have been made on the stress and strain fields ahead of the crack tip for ideally-plastic and power-law hardening materials. The $J$ integral for plane cracks is widely exploited for studying fracture in both linear and non-linear elastic materials, through which a approximate solution of plastic zone for ideally-plastic material was concluded combined with the slip line field analysis (Rice, 1968). Pan and Shih $(1986,1988)$ obtained the crack-tip fields for power-law hardening orthotropic materials. These fields are of the Hutchinson-Rice-Rosengren (HRR) type, and the deformations of these fields are volume preserving. An example of the HRR type crack-tip fields with volumetric deformation was presented by Hutchinson (1983) for power-law creep materials undergoing creep-constrained grain boundary cavitation. As for pressure-sensitive dilatant materials, Li and Pan (1990a,b) analyzed the crack-tip stress and strain fields under 2D plane stress and strain conditions, by introducing a hydrostatic stress-dependent yield criterion and the normality flow rule. They also obtained mode I crack-tip fields for power-law hardening materials within a limited degree of pressure sensitivity. Based on the Drucker-Prager yield criterion, Jeong et al. (1994) constructed theoretical slip lines in front of a round notch tip in a pressure sensitive material. Basu and Van der Giessen (2002) conducted a finite deformation analysis of crack tip fields in glassy polymers using a viscoplastic constitutive model that incorporated softening as well as orientation hardening. For pressure sensitive plastic solids, Subramanya et al. (2007) performed a 3D finite element analysis of mode I crack tip fields under small-scale yielding (SSY) conditions. The crack tip for MGs was studied by Tandaiya et al.(2007, 2008), by using a continuum elastic-viscoplastic constitutive theory developed by Anand and Su (2005), and it was found that these features of plastic field, in turn, are influenced by the mechanical characteristics of MGs like Poisson's ratio and pressure sensitivity. Henann and Anand (2009) conducted finite-element simulations of fracture initiation at notch tip in a MG under mode I, plain-strain, SSY conditions, and revealed the correlations of the fracture toughness with notch-tip radius and elastic modulus. Combining a simple version of the STZ model with an advanced Eulerian level set formulation, Rycroft and Bouchbinder (2012) analyzed the crack tip behavior of a blunted straight notch under plane-strain condition in MGs. They proposed that the existence of the elastoplastic crack tip instability leads to a marked drop in the fracture toughness. These pioneer works provide an important foundation for a better understanding of the crack tip fields and the resultant fracture behavior.

The variability in the fracture toughness or plasticity of MGs is found to be the nature of processes occurring near the crack tip such as shear banding and crack branching, and thus results in different characteristic fracture morphologies (Lewandowski et al., 2005; Xi et al., 2005; Wang et al., 2006; Tandaiya et al., 2009; Xu et al., 2010; Ritchie, 2011; Wu et al., 2011; He et al., 2012; Narayan et al., 2014). From brittle MGs (e.g. Fe- and Mg-based) to ductile ones (e.g. Zr- and Pdbased), the fracture toughness can vary from $\sim 1 \mathrm{~s} \mathrm{MPam}^{1 / 2}$ to $100 \mathrm{~s} \mathrm{MPam}^{1 / 2}$ (Lewandowski et al., 2005; Demetriou et al., 2011; Xu and Ma, 2014). The crack initiation in brittle MGs is revealed to be stress controlled and involves cavity nucleation in front of the crack tip (Narayan et al., 2014). As is proposed that the cavitation stress and the hydrostatic stress ahead of crack tip determine whether brittle fracture via cavitation occurs or not in MGs (Singh et al., 2013, 2014). A misty-mirror morphology presents on the fracture surface of brittle MGs. Nanoscale patterns like dimple and periodic corrugation have been observed in the featureless mirror area (Xi et al., 2005, 2006), which indicates that even for MGs with ideal brittle behavior, the fracture still proceeds by a local softening mechanism but at different length scales. Xi et al. (2005) reported the dimple structures due to the highly localized strain and built a correlation between fracture toughness and plastic zone size. The quasi-brittle corrugation patterns are revealed to be the result of a random creation of cavities or voids in microscale (Xi et al., 2006; Wang et al., 2007). In contrast, the failure in ductile MGs is generally controlled by strain (Flores and Dauskardt, 2006), and the plastic zone accommodating plastic deformation around the crack tip exerts the most direct influence on it. The fracture surface in ductile MGs usually displays vein-like patterns. This typical pattern is ascribed to a Taylor instability process of a fluid meniscus (Spaepen, 1975; Argon and Salama, 1976). The stress softening caused by free volume creation and temperature rise is regarded as the physical reason for yielding and shear banding in MGs (Dai et al., 2005; Jiang and Dai, 2009; Dai, 2012; Greer et al., 2013), and it is naturally a major factor in the evolution of plastic zone. The experimental 
and simulation descriptions of plastic zone in MGs provide important information about potential deformation and fracture mechanisms. However, a quantitative determination of plastic zone is largely ambiguous for MGs whose plastic deformation or nonliearity is extremely localized to micrometer and even nanometer scale. Therefore, a theoretical analysis of the plastic zone ahead the crack tip is significant and urgently required for these materials.

Plastic zones broadly exist in deformed MGs, their stress and strain distributions, sizes and shapes vary largely from ductile to brittle MGs, which leads to distinct deformation modes via fracture toughness and surface morphology. To this end, two questions naturally arise. How to quantitatively characterize the plastic zone ahead of the crack tip in MGs of different ductilities? Most importantly, what's the physical picture of the correlation between the crack-tip plastic zone and the intrinsic ductility of MGs? To answer these questions, we undertake a detailed analysis of the plastic zone for mode I crack under plain strain condition in MGs where the effects of pressure-sensitivity, dilatancy, and structural evolution are included. This paper is organized as follows. In Section 2, a theoretical model is developed to analyze the plastic zone in front of crack tip for MGs through slip line field analysis and $J$-integral, where a pressure-sensitive yield criterion and a new function of plastic zone shape is taken into account. In Section 3, the plastic zone size and shape are discussed. Two characteristic length scales and their ratio are found to be involved with the deformation and fracture behavior, where the influences of Poisson ratio, pressure sensitivity, and dilatancy are elucidated. The stress and the strain distributed in the plastic zone are presented in Section 4. In Section 5, the critical stress intensity factor and the critical plastic zone at crack initiation are analyzed, the internal correlation between fracture pattern and plastic zone is found out and the underlying ductile-to-brittle transition mechanism from the viewpoint of plastic zone is revealed. Finally, the main conclusions of this work are summarized in Section 6.

\section{Theoretical model}

In describing the plasticity of conventional crystalline alloys, it is generally assumed that plastic yielding is independent of the hydrostatic stress and plastic dilatancy is negligible. Several classical theories related to crack field have been initially carried out based on pressure-insensitive von Mises yield criterion and plastic incompressibility, such as the well-known Prandtl slip line field solutions around a sharp crack tip provided by Rice (1968). However, pressure-sensitivity and shear dilatancy cannot be ignored in disordered solids, and the normality plastic flow rule is not always valid for these materials. The pressure sensitivity and dilatancy were then introduced into the slip lines analysis of the sharp crack tip by Li and Pan (1990a,b) and a round notch tip by Jeong et al. (1994). In addition to these commonness shown in disordered materials, the plastic field ahead of the crack tip in MGs experiences a material softening due to thermal or free volume effect, and the evolution of structural disorder (characterized by free volume) has been found to play a dominant role on material instability (Jiang and Dai, 2009). For a deep understanding of the plasticity in MGs, we focus on the nano- or micro-scale plastic zone ahead of a sharp crack tip under mode I loading as illustrated in Fig. 1(a). For a plane strain case, we attempt to establish a theoretical model of plastic zone through $J$-integral based on slip line field analysis and shape approximation, by taking pressure sensitivity, shear dilatancy, and structural evolution into account.

\subsection{Constitutive relations}

There are several models used for MGs to characterize their yield behavior. These include the traditional von Mises model, the Mohr-Coulomb model, the pressure-modified Tresca model, the Drucker-Prager model, and the newly developed yield criterion by Sun et al. (2010b) who incorporated pressure sensitivity correlating to dilatation, temperature dependence and atomic deformation units. Easy to find that, most of these models take the normal stress or pressure into account. Experiments and molecular statics simulations on MGs indicate that the normal or hydrostatic as well as the shear stress acting on the slip plane influence their yield behavior, which is accurately captured by the normal stress or pressure-dependent criterion (Flores and Dauskardt, 2001; Schuh and Lund, 2003; Schuh et al., 2007; Fornell et al., 2009). Here we adopt a pressuresensitive yield criterion analogous to the linear Drucker-Prager model to describe the deformation of MGs, which is expressed as

$$
\Phi=\tau_{e}+\mu \sigma_{m}=\tau_{y},
$$

where the von Mises effective stress $\tau_{e}=\left(s_{i j} s_{i j} / 2\right)^{1 / 2}$, the deviatoric stress $s_{i j}=\sigma_{i j}-\sigma_{m} \delta_{i j}$, the mean stress $\sigma_{m}=\sigma_{k k} / 3$ and the material constant $\mu$ measures the pressure sensitivity of yielding. The yield strength $\tau_{y}$ depends on the structural disorder, i.e. the content of free volume. The more free volume exists, the more significant the stress softening is. Here a simple reasonable form $\tau_{y}=\tau_{0}\left[1-q\left(\xi-\xi_{0}\right)\right]$ is taken, according to these expressions developed by Jiang and Dai (2011) and Chen et al. (2013). $\xi_{0}$ is the initial free volume concentration which can be defined as $\xi_{0}=\left[T-T_{\text {ref }}+\sqrt{\left(T-T_{r e f}\right)^{2}+d_{2} T}\right] / 2 d_{1}$ using the Cohen-Grest model (Grest and Cohen, 1981; Yang et al., 2006), where $T$ is the temperature, $d_{1}, d_{2}$, and $T_{\text {ref }}$ are material parameters. $\xi$ is the current free volume concentration, and the evolution of $\xi$ relies on applied stress, heat and diffusion (Spaepen, 1977; Argon, 1979; Huang et al., 2002; Thamburaja and Ekambaram, 2007; Li et al., 2013). $q$ is the free volume softening coefficient. $\tau_{0}$ is the yield strength of the initial structure (i.e. $\xi=\xi_{0}$ ). 

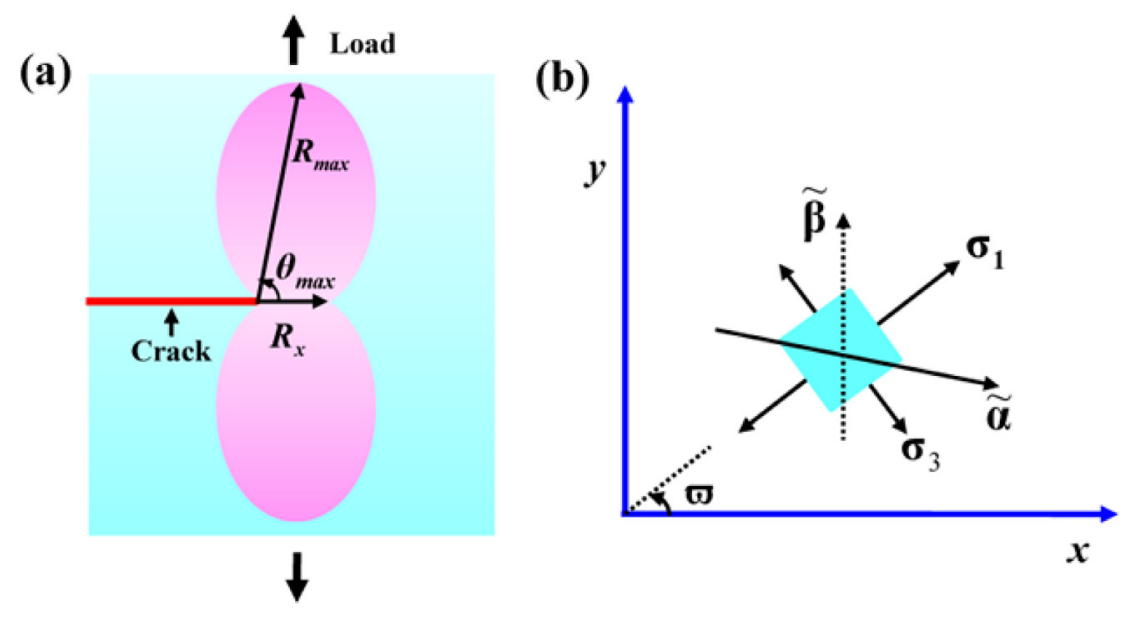

(c)

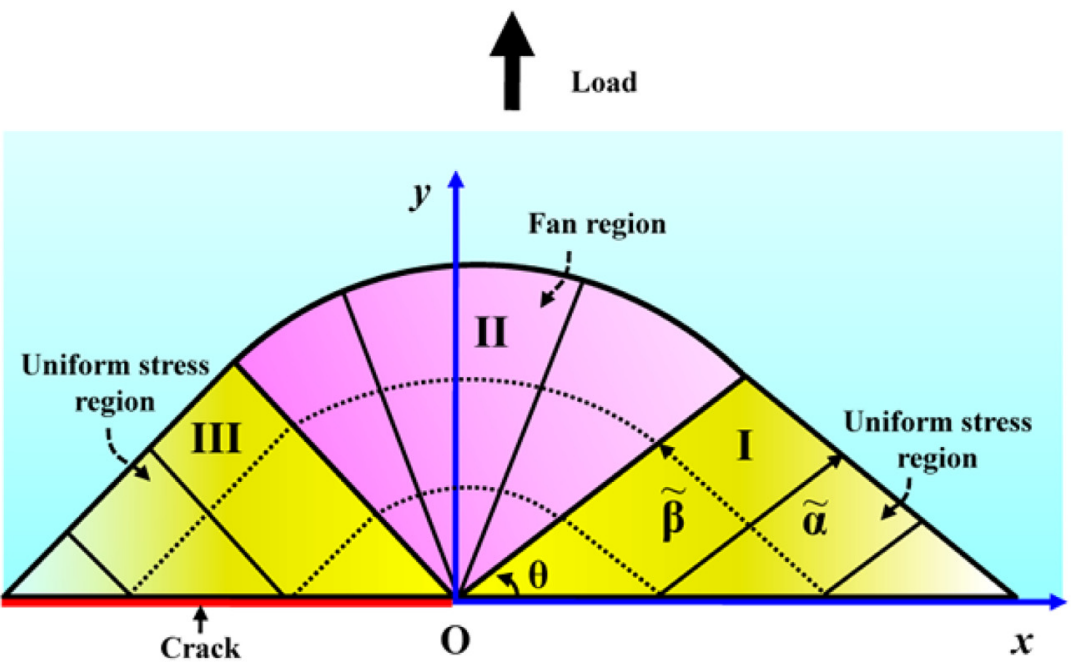

Fig. 1. (a) Illustration of plastic zone in front of a mode I crack tip, (b) slip lines $\tilde{\alpha}$ and $\tilde{\beta}$ and the major principal stresses $\sigma_{1}$ and $\sigma_{3}$ in the $x$-y plane, and (c) slip line field ahead of the mode I crack tip.

The plastic flow of MGs occurs as a consequence of formations and self-organizations of flow events, which are essentially local rearrangements of atoms around free volume sites, termed STZs (Argon, 1979; Falk and Langer, 1998; Johnson and Samwer, 2005; Sun et al., 2010a; Zhao et al., 2013). This local rearrangement involves not only the displacement of atoms but also the evolution of free volume. Under the applied stress, the structural disorder characterized by free volume is evolved, and it is assumed to follow the model of Argon (1979) and Li et al. (2013):

$$
\dot{\xi}=2 \varepsilon_{v}(1-\xi) v_{S T Z} \exp \left(-\frac{\Delta F_{S T Z}(\xi)}{k T}\right) \cosh \left(\frac{\tau \gamma_{0} \Omega_{0}}{2 k T}\right)-2 \varepsilon_{v} \xi(1-\xi) v_{D} \exp \left(-\frac{\Delta G_{D}(\xi)}{k T}\right) .
$$

In Eq. (2), the first term on the right hand denotes the free volume creation via STZ activation, $\varepsilon_{v}$ is the local transformation dilatation, $v_{S T Z}$ and $v_{D}$ are respectively the attempt frequency along the reaction pathway and the frequency factor for diffusive rearrangement, both of which are on the order of the Debye frequency, $k$ is Boltzmann's constant, and $\Omega_{0}$ denotes the STZ volume. The activation barrier for shearing an STZ is expressed as $\Delta F_{S T Z}(\xi)=\Delta F_{\text {shear }}+\left(\Delta F_{D}+\Delta F_{S}\right) \cdot g_{S T Z}(\xi)$, where $\Delta F_{\text {shear }}=\frac{7-5 v}{30(1-v)} G(T) \gamma_{0}^{2} \Omega_{0}$, representing the strain energy associated with an STZ shearing by the characteristic shear strain $\gamma_{0}$, $G(T)$ is the temperature-dependent shear modulus, and $\Delta F_{\text {shear }}$ is independent on free volume. $\Delta F_{D}=\frac{2(1+v)}{9(1-v)} G(T) \varepsilon_{v}^{2} \Omega_{0}$ is the strain energy for a temporary dilatation to allow the atoms to rearrange into the sheared position, and $\Delta F_{S}=\frac{1}{2 \gamma_{0}} \frac{\widehat{\tau}}{G(T)} G(T) \gamma_{0}^{2} \Omega_{0}$ measures the energy required to freely shear an STZ, $\widehat{\tau}$ is the interatomic shear resistance. $g_{S T Z}(\xi)$ is a free-volume dependent term, defined as $g_{S T Z}(\xi)=\left\{1+\alpha\left[\varepsilon_{v}(1-\xi)\right]^{2}\right\} /\left(1+\alpha \varepsilon_{v}^{2}\right)$ with a proportionality factor $\alpha(\sim 0.8)$. The activation energy required to trigger a single event of excess free volume annihilation is expressed as $\Delta G_{D}(\xi)=\Delta G_{D 0} \cdot(1-\xi)$, where $\Delta G_{D 0}$ is the activation free energy for a net diffusive rearrangement in a region with $\xi=0$, and it is taken to be equal to the excess free-volume- 
dependent part of STZ activation energy, i.e. $\Delta G_{D 0}=\Delta F_{D}+\Delta F_{S}$. For the mode I cracking, the shear stress $\tau$ can be expressed by $\tau=K_{I} / L$, where $L$ is a characteristic length relating to the crack size and geometry.

Considering the shear dilatancy inherent in the deformation of MGs, we introduce the dilatancy factor $\beta$, which serves as the ratio of plastic volumetric strain rate $\dot{\varepsilon}_{k k}^{p}$ to the effective plastic shear strain rate $\dot{e}_{e}^{p}$, i.e. $\beta=\dot{\varepsilon}_{k k}^{p} / \dot{e}_{e}^{p}$ (Rudnicki and Rice, 1975). $\Psi$ is invoked as the plastic potential as below

$$
\Psi=\tau_{e}+\beta \sigma_{m},
$$

where the dilatancy factor $\beta$ is not necessary to equal to the pressure sensitivity factor $\mu$. If $\beta=\mu$, the plastic strain obeys the plastic normality rule, otherwise, it follows the plastic non-normality rule. From Eq. (3), the resulting relation between the stresses and plastic strain rates is

$$
\dot{\varepsilon}_{i j}^{p}=\dot{\lambda} \partial \Psi / \partial \sigma_{i j}=\dot{\lambda}\left(s_{i j} / 2 \tau_{e}+\beta / 3 \cdot \delta_{i j}\right),
$$

where $\dot{\lambda}=\dot{e}_{e}^{p}$. When the elastic strains are assumed to be negligible compared with the plastic strains, the plain strain condition requires that,

$$
\dot{\varepsilon}_{33}^{p}=\dot{e}_{e}^{p}\left(s_{33} / 2 \tau_{e}+\beta / 3\right)=0,
$$

and then, we have

$$
\tau_{e}=\left(1-\beta^{2} / 3\right)^{-1 / 2}\left[\left(\frac{\sigma_{11}-\sigma_{22}}{2}\right)^{2}+\sigma_{12}^{2}\right]^{1 / 2}
$$

and

$$
\Phi=\frac{1-\mu \beta / 3}{\sqrt{1-\beta^{2} / 3}}\left[\left(\frac{\sigma_{11}-\sigma_{22}}{2}\right)^{2}+\sigma_{12}^{2}\right]^{1 / 2}+\mu \cdot \frac{\sigma_{11}+\sigma_{22}}{2} .
$$

In the polar coordinates, the yield condition is expressed by

$$
\Phi=\frac{1-\mu \beta / 3}{\sqrt{1-\beta^{2} / 3}}\left[\left(\frac{\sigma_{r r}-\sigma_{\theta \theta}}{2}\right)^{2}+\sigma_{r \theta}^{2}\right]^{1 / 2}+\mu \cdot \frac{\sigma_{r r}+\sigma_{\theta \theta}}{2}=\tau_{y} .
$$

The crack-tip fields can be obtained by solving the two equilibrium equations together with the yield condition for the three unknown stress components, $\sigma_{r r}, \sigma_{\theta \theta}$, and $\sigma_{r \theta}$. To enable an analysis of the slip line field, Eq. (8) can be revised into the Mohr-Coulomb yield criterion by introducing two parameters, i.e. the angle of internal friction $\phi$ and the cohesion $c$, that is

$$
\tau+\tan \phi \cdot \sigma=\left[\left(\frac{\sigma_{r r}-\sigma_{\theta \theta}}{2}\right)^{2}+\sigma_{r \theta}^{2}\right]^{1 / 2} \cdot \frac{1}{\cos \phi}+\tan \phi \cdot \frac{\sigma_{r r}+\sigma_{\theta \theta}}{2}=c
$$

where $\sigma$ and $\tau$ are respectively the normal stress and the shear stress on the yield plane. $\phi$ and $c$ satisfy

$$
\tan \phi=\frac{\mu \sqrt{1-\beta^{2} / 3}}{\sqrt{1-2 \mu \beta / 3-\mu^{2}+4 \mu^{2} \beta^{2} / 9}}
$$

and

$$
c=\frac{\tau_{y} \sqrt{1-\beta^{2} / 3}}{\sqrt{1-2 \mu \beta / 3-\mu^{2}+4 \mu^{2} \beta^{2} / 9}} .
$$

The yield condition in the above form allows us to use the slip line theory based on the Mohr-Coulomb criterion which is usually applied in soil and concrete mechanics (Wu, 1966; Nielsen, 1984). 


\subsection{Slip line field solutions}

Slip line field theory can provide analytical solutions of plastic deformation (i.g. stress field) ahead the crack tip in plane strain state for rigid-plastic solids. There will always be two intersectant directions of maximum shear stress in a plane (see Fig. 1(b)). These generate a curvilinear mesh of two families of slip lines, which always cross each other at right angles. One set of lines are named $\tilde{\alpha}$ lines (solid lines), and the other are called $\tilde{\beta}$ lines (dotted lines).The stress and velocity distribution in material can always be determined from the geometry of these lines. In front of the crack tip, the field consists of two constant stress zones (regions I and III) and a centered fan zone (region II), as shown in Fig. 1(c), where an upper part of the model is depicted due to symmetry. Based on Eq. (9), the complete crack-tip stress fields expressed in the polar coordinates are obtained as

$$
\sigma_{i i}=c \cdot \tilde{\sigma}_{i i}(\phi, \theta)
$$

The expressions of $\tilde{\sigma}_{i i}(\phi, \theta)$ respectively for the regions I,II, and III are shown in detail in Appendix A. From Eq. (4), since the value of $s_{i j} / 2 \tau_{e}$ is independent of time, the plastic strain can be written as

$$
\varepsilon_{i j}^{p}=e_{e}^{p}\left(s_{i j} / 2 \tau_{e}+\beta \delta_{i j} / 3\right),
$$

where the effective plastic shear strain $e_{e}^{p}=\sqrt{2 e_{i j}^{p} e_{i j}^{p}}$ with the deviatoric strain $e_{i j}^{p}=\varepsilon_{i j}^{p}-\varepsilon_{k k}^{p} \delta_{i j} / 3$. Applying Eq. (12) into (13) yields the following stress-strain relation under plane-strain conditions,

$$
\begin{aligned}
\varepsilon_{r r}^{p} & =e_{e}^{p}\left[\left(\sigma_{r r}-\sigma_{\theta \theta}\right) / 4 \tau_{e}+\beta / 2\right], \\
\varepsilon_{\theta \theta}^{p} & =e_{e}^{p}\left[\left(\sigma_{\theta \theta}-\sigma_{r r}\right) / 4 \tau_{e}+\beta / 2\right], \\
\varepsilon_{r \theta}^{p} & =e_{e}^{p}\left[\tau_{r \theta} / 2 \tau_{e}\right] .
\end{aligned}
$$

\subsection{Shape approximation of plastic zone}

In MGs, the plastic deformation is extremely localized to micrometer and even nanometer scale. For a sharp crack inside a MG, the yielded zone at the tip is usually small compared to characteristic geometric dimensions such as notch length. The situation can be treated as "small-scale yielding", in which linear elastic stress-intensity factors form a useful measure of the intensity of the surrounding elastic field.

Based on the linear elastic fracture mechanics, the initial yield radius of the crack tip field is expressed by (detailed in Appendix B)

$$
r_{y}=\frac{1}{2 \pi}\left(\frac{K_{I}}{\tau_{y}}\right)^{2} g(\theta)
$$

with

$$
g(\theta)=\cos ^{2} \frac{\theta}{2}\left\{\sin ^{2} \frac{\theta}{2}+\frac{1}{3}(1-2 v)^{2}+\frac{4 \mu^{2}}{9}(1+v)^{2}+\frac{4 \mu}{3}(1+v)\left[\sin ^{2} \frac{\theta}{2}+\frac{1}{3}(1-2 v)^{2}\right]^{1 / 2}\right\} .
$$

With the load increasing, the plastic zone should grow. Here we assume that under the condition of small scale yielding, the elastic-plastic boundary expands isotropically and the shape of plastic zone is always similar to that of the initial yielding, i.e. $R(\theta) \propto g(\theta)$. Therefore, the plastic zone radius can be divided into the angle-independent term $R$ and the angle-dependent term $g(\theta)$, in the form of

$$
R(\theta)=R \cdot g(\theta)
$$

In the plastic zone, there are two characteristic lengths as shown in Fig. 1(a). One is the maximum plastic zone radius $R_{\text {max }}$ which measures the largest distance from the crack tip to the elastic-plastic boundary. The angle corresponding to $R_{m a x}$ yields $\partial g(\theta) / \partial \theta=0$, and it is derived as

$$
\theta_{\max }=2 \arcsin \left(\sqrt{\frac{B-\sqrt{B^{2}-4 C}}{2}}\right)
$$

where $B=\mu^{2}(1+v)^{2} / 9-(1-2 v)^{2} / 3+1$, and $C=\left[(1-2 v)^{2} / 6-0.5\right]^{2}-\mu^{2}\left(1-v-2 v^{2}\right)^{2} / 27$. 
The other length is the radius along the crack line direction, i.e. $R_{x}$, satisfying that $\theta=0$. The ratio $\Lambda=R_{x} / R_{\max }$ is defined as the shape factor of the plastic zone.

\subsection{Solution of plastic zone from J-integral}

According to Rice (1968), the path-independent J-integral can be introduced. The proof of path independence applies strictly to paths surrounding the notch tip in elastic material outside the plastic zone. Path independence results are also valid for paths passing through the plastic zone when a deformation plasticity theory, which is really nonlinear elasticity, is employed. The $J$-integral is expressed as

$$
J=\int_{\Gamma}\left(W d x_{2}-\mathbf{T} \cdot \partial \mathbf{u} / \partial x_{1} d s\right)
$$

with

$$
W=\int_{0}^{\varepsilon} \sigma_{i j} d \varepsilon_{i j}
$$

and

$$
\mathbf{T} \cdot \partial \mathbf{u} / \partial x_{1}=\sigma_{r r}\left(\frac{\partial u_{r}}{\partial r} \cos \theta-\frac{\partial u_{r}}{\partial \theta} \frac{\sin \theta}{r}+u_{\theta} \frac{\sin \theta}{r}\right)+\tau_{r \theta}\left(\frac{\partial u_{\theta}}{\partial r} \cos \theta-\frac{\partial u_{\theta}}{\partial \theta} \frac{\sin \theta}{r}-\frac{u_{r}}{r} \sin \theta\right)
$$

where $W$ denotes the energy density of material, $\mathbf{T}$ is the traction vector on a defined path according to the outward normal, $\mathbf{u}$ is the displacement vector, $\Gamma$ is the arbitrary path surrounding the tip and is traversed in the contra-clockwise sense, and $s$ is arc length.

The energy line integral $J$ of Eq. (19) can be evaluated on a circle of radius $r$ surrounding the crack tip, and path independence permits us to let $r \rightarrow 0$. We define $u_{\theta}=f(\theta)+g(r)$, when it is very near the crack tip $r \rightarrow 0, g(r)=0$. Then $\partial u_{\theta} / \partial r=0$. In view of the strain boundary (Eq. (17)), all the three regions (the constant stress regions I and III, and the fan region II) make contributions to J. Employing the displacements and strains, one finds that, Eq. (19) results in

$$
J=2 \sum_{k=I, I I, I I I} \int_{\theta_{1}^{k}}^{\theta_{2}^{k}}\left[\left(\tau_{r \theta}^{k} \gamma_{r \theta}^{k}+\sigma_{\theta \theta}^{k} \varepsilon_{\theta \theta}^{k}\right) \cos \theta+\left(\sigma_{r r}^{k} \gamma_{r \theta}^{k}+\tau_{r \theta}^{k} \varepsilon_{\theta \theta}^{k}\right) \sin \theta\right] r d \theta
$$

Assuming that the effective plastic strain follows $e_{e}^{p}=e_{0}^{p} \cdot R(\theta) / r$ similar to the form proposed by Rice (1968), we have

$$
J=2 \sum_{k=I, I I, I I I} \int_{\theta_{1}^{k}}^{\theta_{2}^{k}}\left[\left(\tau_{r \theta}^{\mathrm{k}} \tilde{\gamma}_{r \theta}^{\mathrm{k}}+\sigma_{\theta \theta}^{k} \tilde{\varepsilon}_{\theta \theta}^{k}\right) \cos \theta+\left(\sigma_{r r}^{k} \tilde{\gamma}_{r \theta}^{k}+\tau_{r \theta}^{k} \tilde{\varepsilon}_{\theta \theta}^{k}\right) \sin \theta\right] R(\theta) e_{0}^{p} d \theta,
$$

where $\tilde{\gamma}_{r \theta}^{k}=\gamma_{r \theta}^{k} / e_{e}^{p}$ and $\tilde{\varepsilon}_{\theta \theta}^{k}=\varepsilon_{\theta \theta}^{k} / e_{e}^{p}$ are normalized strains, and $e_{0}^{p}=c / G . \theta_{1}^{I}=0, \theta_{2}^{I}=\theta_{1}^{I I}=\pi / 4-\phi / 2, \theta_{2}^{I I}=\theta_{1}^{I I I}=3 \pi / 4-\phi / 2$, $\theta_{2}^{I I I}=\pi$, and $R(\theta)$ is expressed by Eq. (17). Combining Eqs. (16), (17), and (21), the value of $R$ can be determined by

$$
R=\frac{J}{2 \sum_{k=I, I I, I I I} \int_{\theta_{1}^{k}}^{\theta_{2}^{k}}\left[\left(\tau_{r \theta}^{k} \tilde{\gamma}_{r \theta}^{k}+\sigma_{\theta \theta}^{k} \tilde{\varepsilon}_{\theta \theta}^{k}\right) \cos \theta+\left(\sigma_{r r}^{k} \tilde{\gamma}_{r \theta}^{k}+\tau_{r \theta}^{k} \tilde{\varepsilon}_{\theta \theta}^{k}\right) \sin \theta\right] g(\theta) e_{0}^{p} d \theta} .
$$

For small scale yielding, the value of $J$ can be approximately taken as the linear elastic crack solution, i.e. $J=\left(1-v^{2}\right) K_{I}^{2} / E$ for plane strain state.

The rate of plastic zone size is then expressed as

$$
\dot{R}=\frac{(1-v)\left(1-2 \mu \beta / 3-\mu^{2}+4 \mu^{2} \beta^{2} / 9\right) /\left(1-\beta^{2} / 3\right)}{2 \sum_{k=I, I I, I I I} \int_{\theta_{1}^{k}}^{\theta_{2}^{k}}\left[\left(\tilde{\tau}_{r \theta}^{k} \tilde{\gamma}_{r \theta}^{k}+\tilde{\sigma}_{\theta \theta}^{k} \tilde{\varepsilon}_{\theta \theta}^{k}\right) \cos \theta+\left(\tilde{\sigma}_{r r}^{k} \tilde{\gamma}_{r \theta}^{k}+\tilde{\tau}_{r \theta}^{k} \tilde{\varepsilon}_{\theta \theta}^{k}\right) \sin \theta\right] g(\theta) d \theta} \cdot\left(\frac{\dot{K}_{I} K_{I}}{\tau_{y}^{2}}+\frac{q \tau_{0} K_{I}^{2} \dot{\xi}}{\tau_{y}^{3}}\right) .
$$

where $\tilde{\tau}_{r \theta}^{k}=\tau_{r \theta}^{k} / c, \tilde{\sigma}_{r r}^{k}=\sigma_{r r}^{k} / c$ and $\tilde{\sigma}_{\theta \theta}^{k}=\sigma_{\theta \theta}^{k} / c$ are normalized stress components. 
The evolution of plastic zone with the free volume under external loading for the mode I cracking can be described by Eqs. (2) and (23). As we mentioned before, the classical J-integral theory relies on the assumption of deformation plasticity which treats elastic-plastic materials as if they were nonlinear elastic. This approach is valid only if the condition of proportional loading is fulfilled. In this work, we focus on the stable evolution process of the plastic zone and the critical point prior to the instability, in which the free volume concentration almost keeps constant or evolves very slowly. Unloading would not occur until the plastic zone loses stability due to a marked increase of free volume and a large drop of flow viscosity. The influences of the material parameters $v, \mu$ and $\beta$ are considered in the behavior of plastic zone. The relationships of these parameters with the fracture toughness and plasticity will be discussed in the following sections. The underlying story behind the ductileto-brittle transition in MGs will be then disclosed.

\section{Plastic zone size and shape}

Due to the stress concentration, materials around the crack tip or flaw should first yield and form a localized plastic zone. The plastic zone will grow with the external loading until the crack runs away. Different from the homogeneous plastic zone in crystalline alloys, the plastic zone in MGs usually firstly experiences minor homogeneous plastic deformation and then develops into an inhomogeneous mode. For different MGs, the sizes of plastic zones can vary from nanometer to micrometer due to their distinct ductilities (Xi et al., 2005; Ashby and Greer, 2006; Yuan et al., 2012). Usually, one length scale, i.e. radius, is used to characterize the size of plastic zone, and it is found that large plastic zone prior to crack presents good plastic flow capability. However, is this single length scale enough to fully describe the whole plastic zone? And besides the size, what role does the plastic zone shape play in the deformation behavior of MGs? Here we make a quantitative description of the plastic zone, including the characteristic lengths and the shape. In the following discussions, the term $(1-2 v)^{2} / 3$ inside the square root bracket of Eq. (16) is neglected in the integration of Eq. (22) to obtain an analytical solution. This simplification is made for several considerations: (i) we note that the fan region (II) makes the major contribution to $J$-integral in contrast to the uniform stress regions (I and III), and (ii) the value of $(1-2 v)^{2} / 3$ is a minor term compared with $\sin ^{2}(\theta / 2)$ (see Eq. (16) in the fan region $(\pi / 4-\phi / 2<\theta<3 \pi / 4-\phi / 2)$ and is vanishing when $v \rightarrow 0.5$. The mechanical and material parameters used in the following analyses are given as (Yang et al., 2006; Li et al., 2013): $v_{S T Z}=v_{D}=1.0193 \times 10^{12} \mathrm{~s}^{-1}, k_{b}=1.38 \times 10^{-23}, \Omega=1.6 \mathrm{~nm}^{3}$, $\varepsilon_{v}=0.01, \gamma_{0}=\varepsilon_{v} / \beta, d_{1}=46981 \mathrm{~K}, d_{2}=162 \mathrm{~K}, T_{r e f}=672 \mathrm{~K}, T=300 \mathrm{~K}, q=1, \tau_{0}=1 \mathrm{GPa}, G=37 \mathrm{GPa}, G_{T}=G-0.004 \mathrm{GPaK}^{-1} \cdot T$, $\widehat{\tau}=G_{T} / 10, \alpha=0.8, \dot{K}_{I}=10^{5} \mathrm{Pam}^{1 / 2} \mathrm{~s}^{-1}$, and the value of $L$ can be estimated of order $100 \mu \mathrm{m}$ for the shear yield stress of order $1 \mathrm{GPa}$ and the $K_{I c}$ value of order $10 \mathrm{MPam}^{1 / 2}$.

It is known that, for typical Zr-based MGs, its Poisson's ratio is around 0.35 and its pressure sensitivity factor $\mu$ and dilatancy factor $\beta$ are estimated about 0.1 (Sun et al., 2010b). Actually, for different MGs, the values of Poisson's ratio can range from 0.3 to nearly 0.5 , and the pressure sensitivity and the dilatancy can change from minor (i.e. $\mu=\beta=0$ ) to significant (normally $\mu$ and $\beta$ are larger than 0.1 ). These variations of material parameters are found to be correlated with the macroductility of MGs. To unravel these underlying mechanisms, we conducted a parametric study of the plastic zone to reveal the variation of the respective effect of $v, \mu$ and $\beta$ on plastic zone. The contours of the plastic zones are presented in Fig. 2, which are plotted in the normalized coordinate $R /\left(K_{I} / \tau_{y}\right)^{2}$. Fig. 2(a) shows the effect of varying Poisson's ratio (i.e. $v=0.3,0.35,0.4,0.45$,and 0.5 ) on the plastic zone for $\mu=\beta=0.1$. It can be seen that with the increase of $v$, the plastic zone size is reduced, and the change is more significant along the leading edge than that along the trailing edge. The dependence of plastic zone on pressure sensitivity and dilatancy is described by Fig. 2(b) for a constant $v=0.35$. For normal plastic flow $(\mu=\beta)$, with increase in $\mu$ and $\beta$, the plastic zone extends markedly; for non-normal plastic flow $(\mu \neq \beta)$, the plastic zone
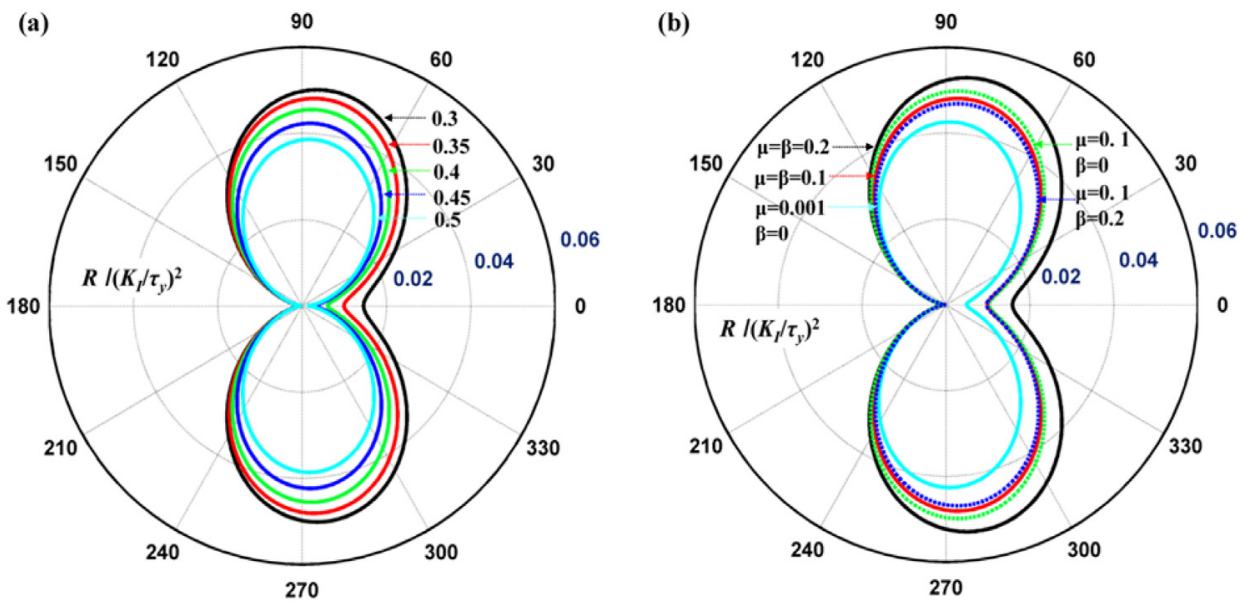

Fig. 2. Contours of plastic zones: (a) for $\mu=\beta=0.1$ and $v=0.3,0.35,0.4,0.45$, and 0.5 , (b) for $v=0.35$ and varied values of $\mu$ and $\beta$. 

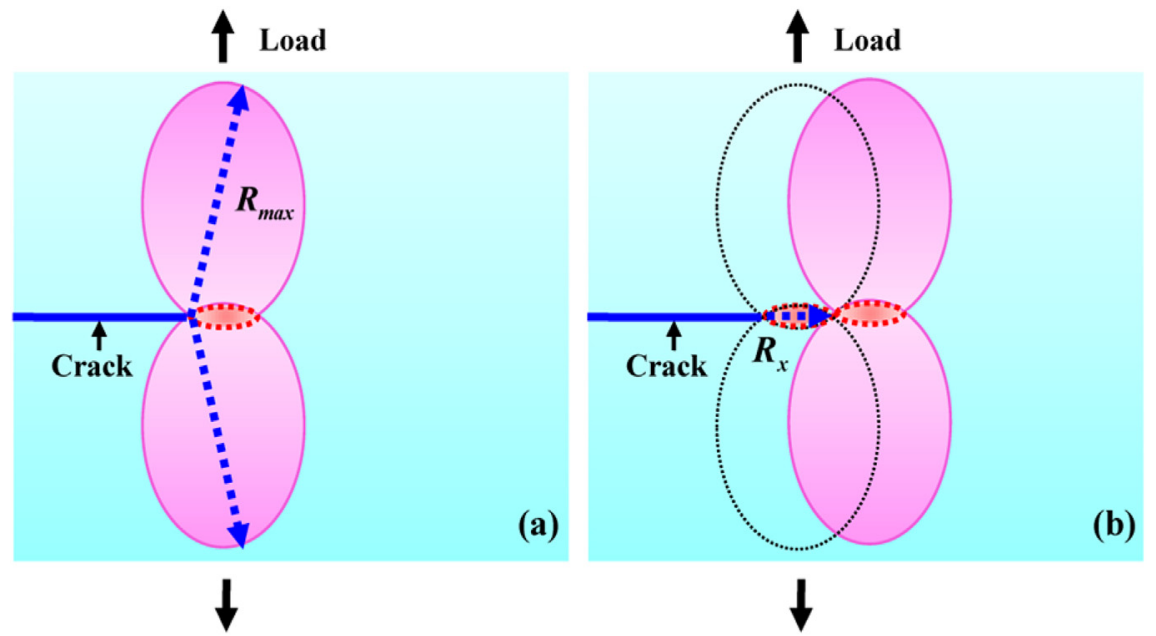

Fig. 3. Illustration of the potential failure modes ahead the crack tip: (a) plastic flow along $R_{\max }$ and (b) cavitation instability along $R_{x}$.

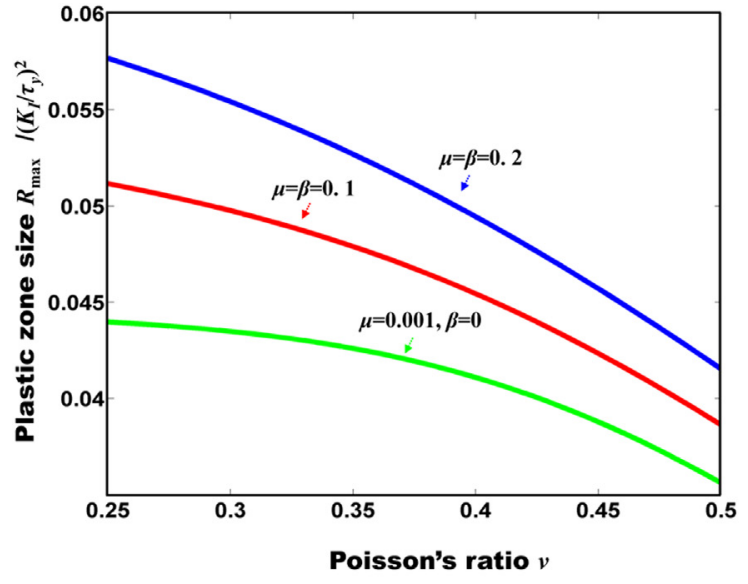

(a)

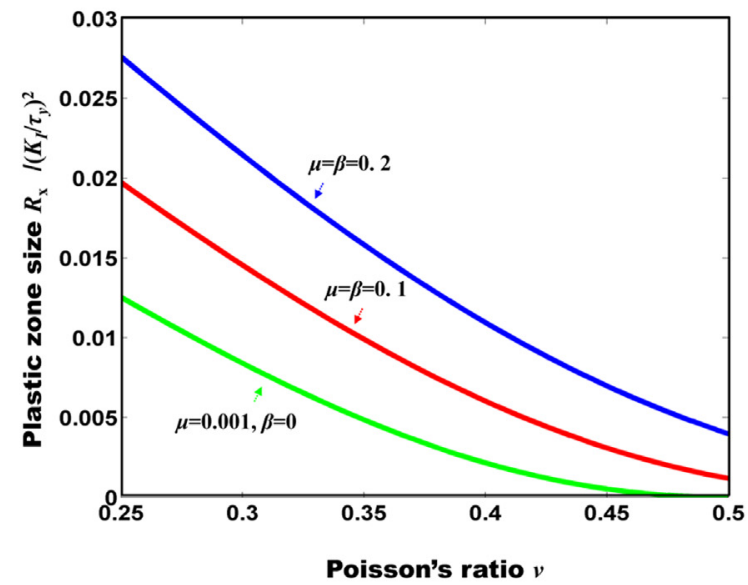

(b)

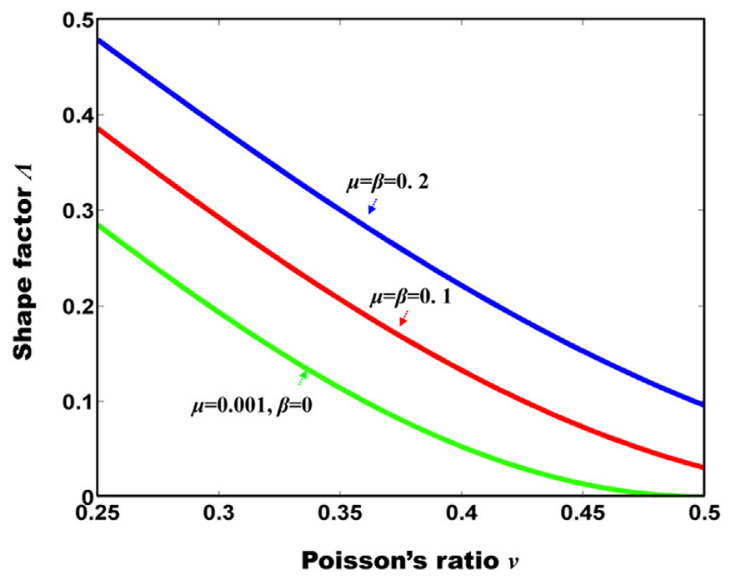

(c)

Fig. 4. Dependences of $R_{\max }(\mathrm{a}), R_{x}$ (b), and the shape factor $\Lambda$ (c) on Poisson's ratio for varied values of $\mu$ and $\beta$. 
reduces gently with $\beta$ increasing from 0 to 0.2 for $\mu=0.1$. The general effects of Poisson's ratio and pressure sensitivity on the plastic zone from our theoretical prediction are consistent with those simulation results reported by Tandaiya et al. (2007, 2008) who conducted the finite element simulation on the stationary mode I crack tip fields under plane strain for MGs. Smaller Poisson's ratio or bigger pressure sensitivity corresponds to a larger plastic zone under the precondition of the same $K_{I}$ and $\tau_{y}$, suggesting a faster growth of plastic zone. As mentioned previously, a large plastic zone at crack initiation usually indicates a good plasticity. We note that the plastic zone discussed here is not the critical one prior to cracking but is a process value for a given $K_{I}$. Since the critical plastic zone greatly relies on the fracture toughness and different MGs usually have distinct fracture toughnesses, small Poisson's ratio or big pressure sensitivity, along with a fast development of plastic zone, unnecessarily leads to a large final plastic zone prior to cracking. The fracture toughness and the corresponding critical plastic zone which depend on material properties will be discussed in Section 5.

One radius is usually used to characterize the plastic zone size. However, this single length scale is not enough to describe the whole plastic zone since the zone boundary is not as a regular circle. Actually, along different directions, the radii of the plastic zone are different, among which two important length scales are raised, i.e. the maximum radius $R_{\text {max }}$ and the radius along the crack line direction $R_{\mathrm{x}}$. With the evolution of plastic zone, two typical instabilities may come along. One is the shear flow instability (Pampillo and Reimschuessel, 1974; Argon and Salama, 1976) and the other is the cavity nucleation and coalescence (Jiang et al., 2008a; Huang et al., 2013; Singh et al., 2014), as illustrated in Fig. 3. MGs fracture by the break-up of a crack tip, the zone ahead of the crack tip is highly strained and its viscosity is reduced due to structural evolution. The strainsoftening yields a liquid-like plastic zone or shear band ahead of the crack tip, and the crack front is expected to act as meniscus (Wang et al., 2007; Tandaiya et al., 2013). When an infinitesimal perturbation of this meniscus reaches a critical wavelength $\lambda_{c}$ for meniscus instability, it grows and advances the crack front into the plastic zone, leading to the formation of vein patterns. It is found that whether crack tip meniscus instability develops or not involves the scale of local softening ahead

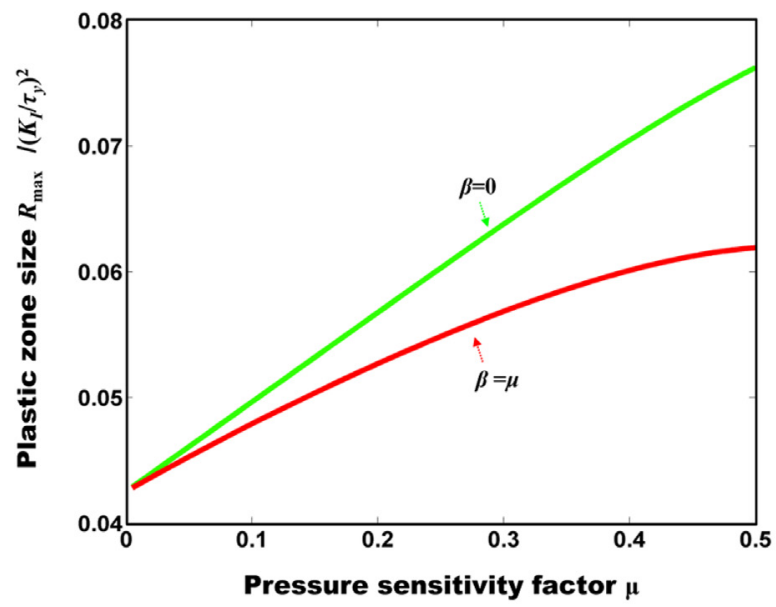

(a)

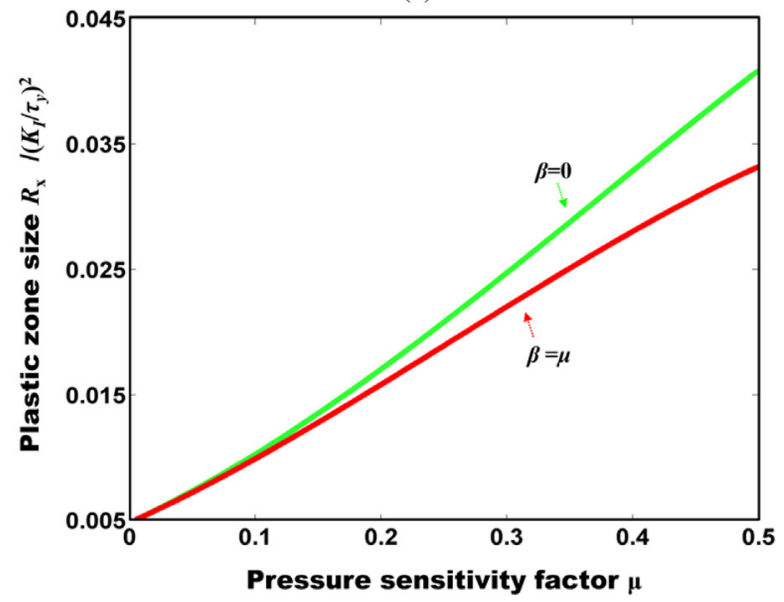

(b)

Fig. 5. Dependences of $R_{\max }(\mathrm{a})$ and $R_{x}$ (b) on Pressure sensitivity factor $\mu$ for $v=0.35, \beta=0$ and $\beta=\mu$ respectively. 
of the crack tip (Wang et al., 2007; Jiang et al., 2008a; Wang et al., 2008; Xia and Wang, 2012). The crack tip meniscus instability develops when the scale of local softening ahead of the crack tip is larger than $\lambda_{c}$, and energy dissipated mainly by local plastic flow (Jiang et al., 2008a). In this sense, the largest distance from the crack front to the plastic zone boundary, characterized by $R_{\max }$, to which the flow meniscus may advance, can be an index of plastic flow ability. When the initial perturbation of the meniscus is restrained, the front of the crack tip maintains a straight line, during which cavitation instability may occur under a high tensile hydrostatic stress. In a narrow region along $R_{X}$ (see Fig. 3(b)), the material is strongly softened and experiences a much larger hydrostatic tensile stress as compared with the other parts within the plastic zone (a detailed discussion is presented in Section 4). Cracking via cavitation prefers to occur in this weak area which behaves as a 'fracture process zone' (Narayan et al., 2014). In this sense, $R_{x}$ measures the potential capability of crack propagation via cavitation. The competition between the two failure modes can be attributed to the competition of the two characteristic lengths, i.e. $R_{\max }$ and $R_{x}$. The shape factor as a ratio of $R_{x}$ to $R_{\max }$, reflects the plastic zone shape and also reveals the potential failure mode.

The variations of the two length scales $R_{\max }$ and $R_{x}$ with Poisson's ratio are respectively plotted in Fig. 4(a) and (b) for different $\mu$ and $\beta$. We can find that, for bigger values of $\mu$ and $\beta$, both $R_{\max }$ and $R_{\mathrm{x}}$ are larger; for the same $\mu$ and $\beta, R_{\max }$ and $R_{\mathrm{x}}$ reduce with increasing Poisson's ratio. The effect of Poisson's ratio on the shape factor $\Lambda$ is illustrated by Fig. 4(c). With the increase of Poisson's ratio, the ratio $\Lambda$ is decreasing, indicating that $R_{\mathrm{X}}$ reduces faster than $R_{\max }$. The large values of $\mu$ and $\beta$ correspond to a big value of $\Lambda$. One can expect that, for a small $\Lambda$ (i.e. large $R_{\max }$ and small $R_{x}$ ), MGs prefer to experience considerable plastic flow and fail through shear flow instability; otherwise, it presents very local softening and fracture via cavity nucleation and coalescence.

The changes of $R_{\max }$ and $R_{\mathrm{X}}$ with the pressure sensitivity factor $\mu$ for $\beta=\mu$ (normal flow) and $\beta=0$ (non-normal flow) are respectively plotted in Fig. 5(a) and (b), in which the Poisson's ratio is taken as a constant value 0.35 . With increase in $\mu$, both $R_{\max }$ and $R_{\mathrm{X}}$ are increasing, and the two lengths for the case of $\beta=0$ is bigger than these for $\beta=\mu$, indicating that non-normal

(a)
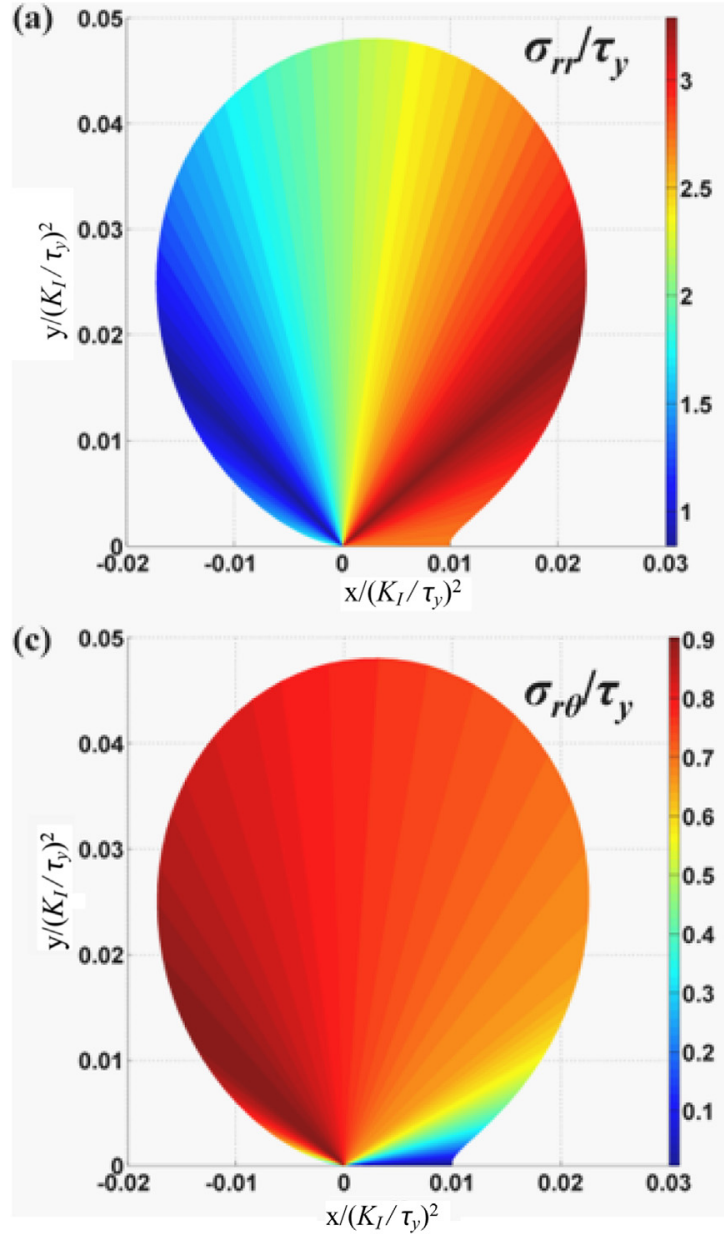

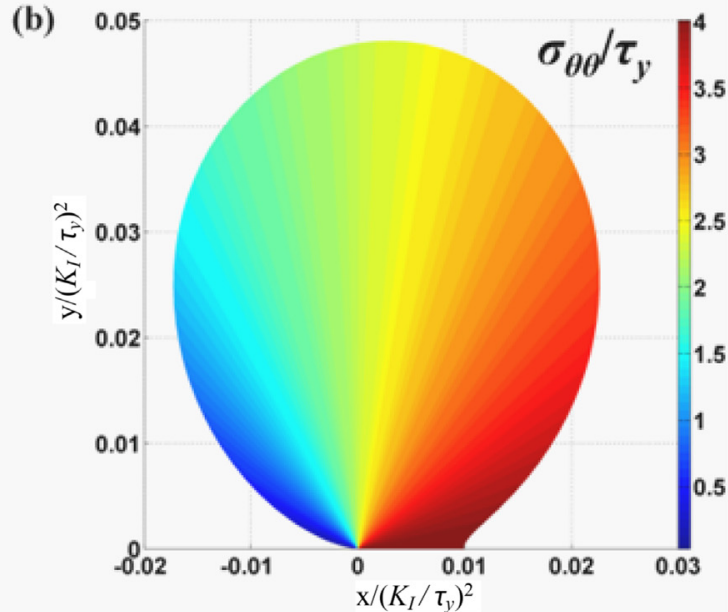

(d)

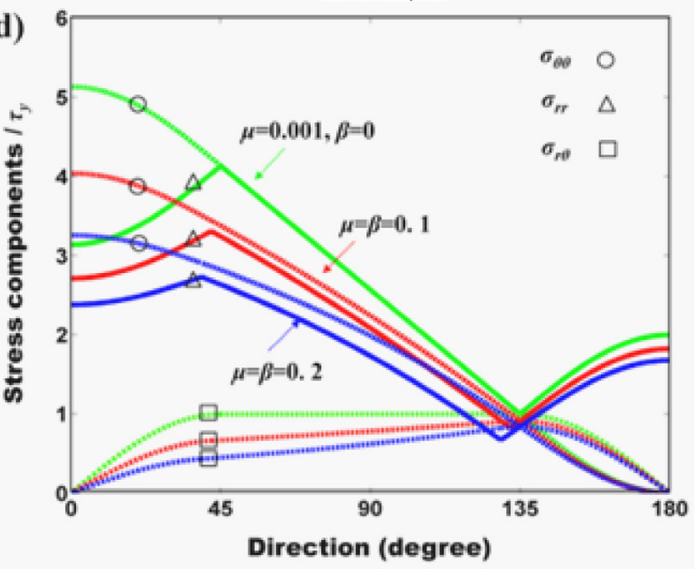

Fig. 6. Contour plots of the distributions of (a) radial, (b) tangential, (c) shear stresses in the plastic zone, and (d) the angular distributions of stress components for different values of pressure sensitivity and dilatancy. 
flow could cause a faster development of plastic zone. This differences of plastic zone between normal flow and non-normal flow are enlarged with the growth of $\mu$.

\section{Stress and strain distribution}

The influences of pressure sensitivity, dilatancy, and Poisson's ratio on the stress and strain fields in front of the crack tip are examined. Fig. 6 shows the angular distributions of (a) radial, (b) tangential, (c) shear stresses normalized by $\tau_{y}$ within the plastic zone for $\mu=\beta=0.1$ and the variation of stresses with angle for different values of pressure sensitivity and dilatancy (d). It can be seen that the radial stress is increasing with the direction angle from $0^{\circ}$ until it reaches the maximum value at a angle around $45^{\circ}$, then it decreases to a minimum value at a angle around $135^{\circ}$, thereafter it grows with the increase of the direction angle. The maximum tangential stress is along the crack line direction and it decreases monotonously with the angle increasing from 0 to $180^{\circ}$. The shear stress first increases and then decreases with the variation of direction angle from 0 to $180^{\circ}$ and the maximum value is along the direction around $135^{\circ}$. From Fig. 6(d), we can find that with the enhancement of pressure sensitivity and dilatancy, all the stress components reduce. A strong pressure sensitivity leads to a substantial decrease in the opening stress. Fig. 7(a) and (b) presents respectively the angular distributions of effective and mean stresses normalized by $\tau_{y}$ for $\mu=\beta=0.1$, and those for different values of pressure sensitivity factor and dilatancy factor are described in Fig. 7(c). The effective stress keeps the minimum and the maximum values respectively in the constant stress regions (I and III), and in the fan region (II) it increases with increasing distribution angle. Oppositely, the mean stress reaches its maximum and minimum respectively in regions I and III, and it decreases with increase in distribution angle. According to these previous works (Murali et al., 2011b; Singh et al., 2013; Singh et al., 2014), when the tensile mean stress exceeds the cavitation stress, microvoid will nucleate and grow. Therefore, region I forms a weak zone facilitating cavitation and cracking. When
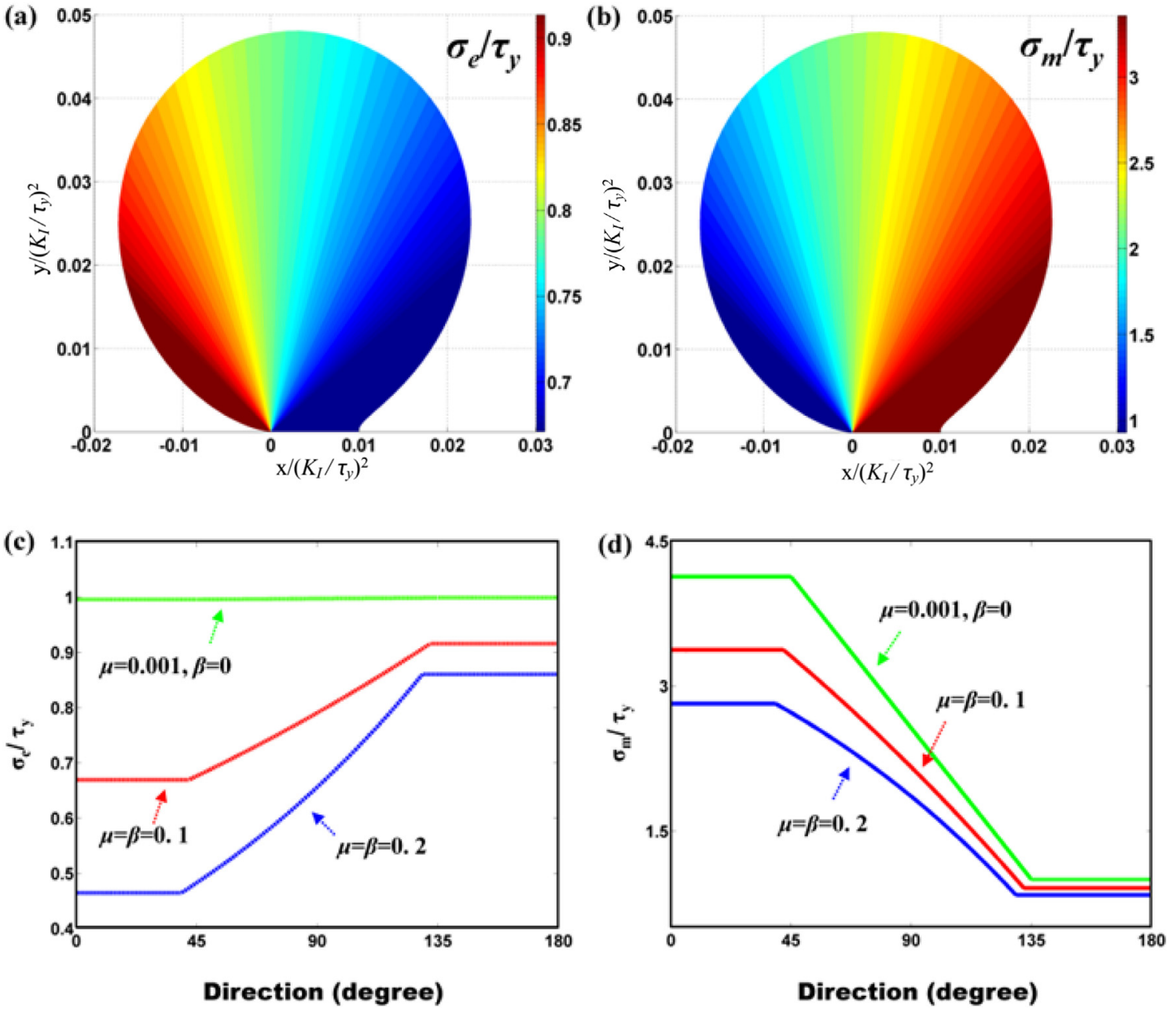

Fig. 7. Contour plots of the distributions of (a) effective, and (b) mean stresses in the plastic zone, and the angular distributions of the effective stress (c) and the mean stress (d) for different values of pressure sensitivity and dilatancy. 
neglecting the pressure sensitivity and dilatancy, the effective stress is a constant value, equal to $\tau_{y}$, in the whole plastic zone. With the increase of $\mu$ and $\beta$, both the effective and the mean stresses decrease, which is consistent with the change trend of stress components.

For perfectly-plasticity crack-tip fields, Li and Pan (1990a) reported that the plastic radial strain $\varepsilon_{r r}$ and tangential strain $\varepsilon_{\theta \theta}$ are near zero and a marked plastic shear strain $\varepsilon_{r \theta}$ is localized into the fan region. As for MGs, considering the pressuresensitivity and dilatancy, the contour plots of plastic radial, tangential, shear strains are respectively shown in Fig. 8(a-c) for $\mu=\beta=0.1$, where $\tau_{y}=1 \mathrm{GPa}$ and $G=37 \mathrm{GPa}$ are introduced. We can see that the radial strain is compressive in region I and is tensile in region III, in the fan region (II) $\varepsilon_{r r}$ is close to zero. The tensile tangential strain is found in regions I and II while the compressive value is in region III. The shear plastic strain almost concentrates in the fan region (II). It is obvious that the shear strain is more significant as compared with the other two. These angular distributions of plastic fields are similar to these results of Li and Pan (1990a) for a pressure-sensitive dilatant material with a power-law hardening exponent of 100 which is close to the perfect-plasticity case.

\section{Critical stress intensity factor and plastic zone}

The plastic zone ahead the crack tip can experience a stable evolution under external loading before the crack initiation. Under the same stress intensity factor, the material with a smaller Poisson's ratio or bigger pressure sensitivity enable a relatively faster growth of plastic zone, as mentioned in section 3. In this stable deformation process, the structural disorder, i.e. free volume, evolves smoothly. Unfortunately, material cannot deform stably all the way. When the applied stress or stress intensity factor reaches a critical value, the free volume will grow rapidly, which causes a severe material softening and an instability of plastic zone. This unstable evolution usually leads to a crack initiation. For different MGs, the critical values of mode I stress intensity factor for instability (as a quantity of fracture toughness) are distinct, it can range from an order of 1-100 MPam ${ }^{1 / 2}$ from the experimental observations (Lewandowski et al., 2005; Xi et al., 2005; Demetriou et al., 2011; Xu and
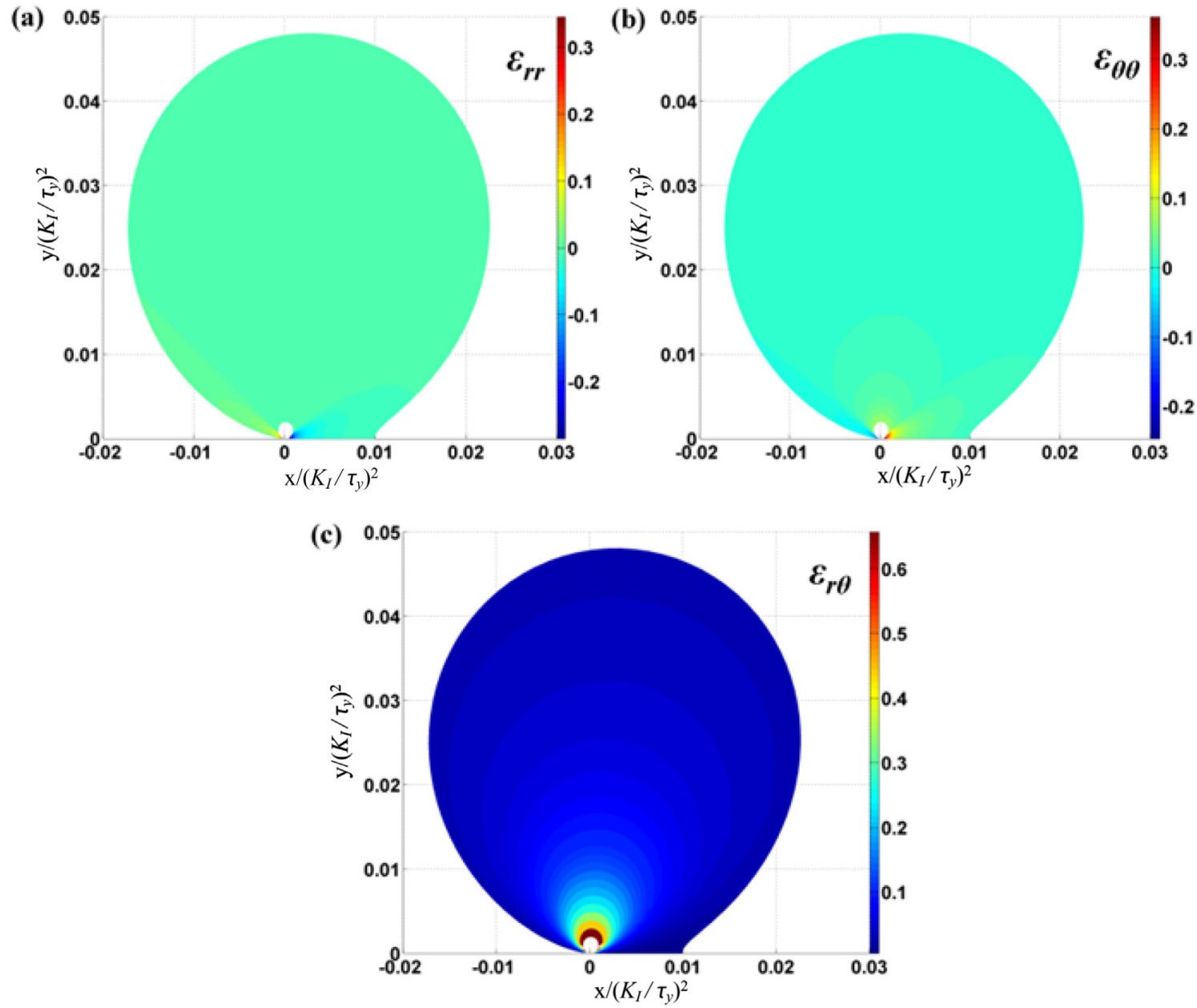

Fig. 8. Contour plots of the distributions of (a) radial, (b) tangential, and (c) shear strains in the plastic zone. 
Ma, 2014). Lewandowski et al. (2005) reported a correlation between fracture toughness and the Poisson's ratio for a variety of MGs and revealed that big Poisson's ratio usually corresponds to a large fracture toughness. The same change trend can be displayed in Fig. 9(a). This plot shows the evolution of free volume with the stress intensity factor for $\mu=\beta=0.1$. In order to reveal the influence of Poisson's ratio $v$ on the free volume evolution, the $v$ values are selected within a reasonable range from 0.3 to 0.5 , and the values of other material parameters required for the analyses in this section accord with those presented in Section 3. It can be seen that, the free volume changes little with the increase of $K_{I}$ before $K_{I}$ reaches a critical value, and thereafter it grows rapidly. We note that the curve for $v=0.35$ is close to a real case of Zr-based MG, and the stress intensity factor at which instability initiates is right consistent with the real fracture toughness (i.e. around $40 \mathrm{MPam}^{1 / 2}$ ) (Lewandowski, 2001; Wang et al., 2002). With the increase of $v$ from 0.3 to 0.5 , the stable-to-unstable transition happens at a larger stress intensity factor, which is in line with that large Poisson's ratio corresponds to high fracture toughness (Lewandowski et al., 2005).

The dependences of the two characteristic lengths of plastic zone on the stress intensity factor are illustrated in Fig. 9(b) and (c). Similar to free volume, both $R_{\max }$ and $R_{x}$ experience a stable-to-unstable transition. We can find that, $R_{\max }$ shows little growth before $K_{I}$ reaches $K_{I c}$, which is true for different values of Poisson's ratio. In contrast, $R_{x}$ displays a considerable increase in the stable stage when Poisson's ratio is small. It is apparent that the magnitude of Poisson's ratio can affect the growth speed of plastic zone, and it has a more significant influence on $R_{x}$ than on $R_{\max }$. With the increase of Poisson's ratio, the critical $R_{\max }$ corresponding to the stable-to-unstable transition almost keeps constant, while the critical $R_{x}$ reduces.

The evolution of free volume with stress intensity factor for $v=0.35$ and different values of pressure sensitivity and dilatancy is presented in Fig. $10(\mathrm{a})$. With $\mu$ and $\beta$ increasing from 0.05 to 0.2 , the critical stress intensity factor reduces markedly from around $67 \mathrm{MPam}^{1 / 2}$ to $17 \mathrm{MPam}^{1 / 2}$. This indicates that in addition to Poisson's ratio, the pressure sensitivity and shear dilatancy should also have a considerable effect on the fracture toughness. For smaller $\mu$ and $\beta$, the plastic zone can experience a longer stable growth and reach a larger size (see Fig. 10(b) and (c)), which may reveal a better ductility. Compared with $R_{\max }, R_{x}$ is much more sensitive to the variations of Poisson's ratio, pressure sensitivity, and dilatancy. As discussed before, $R_{\max }$ and $R_{x}$ are respectively related to the behaviors of plastic flow and cavitation. It probably suggests that

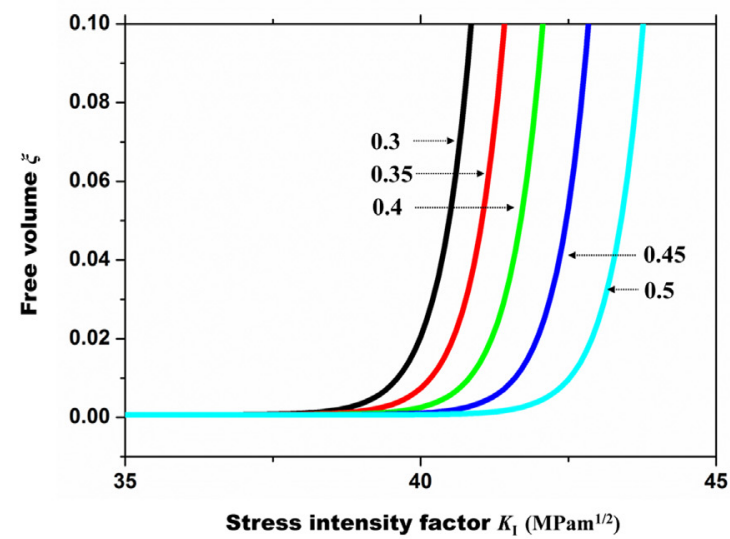

(a)

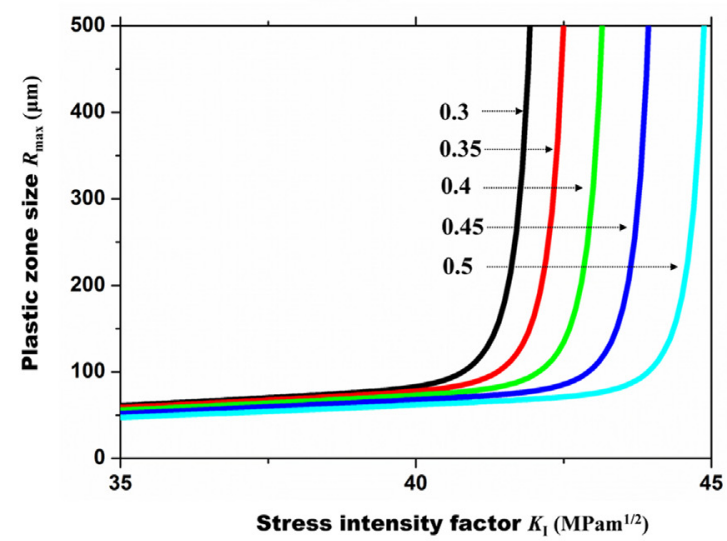

(b)

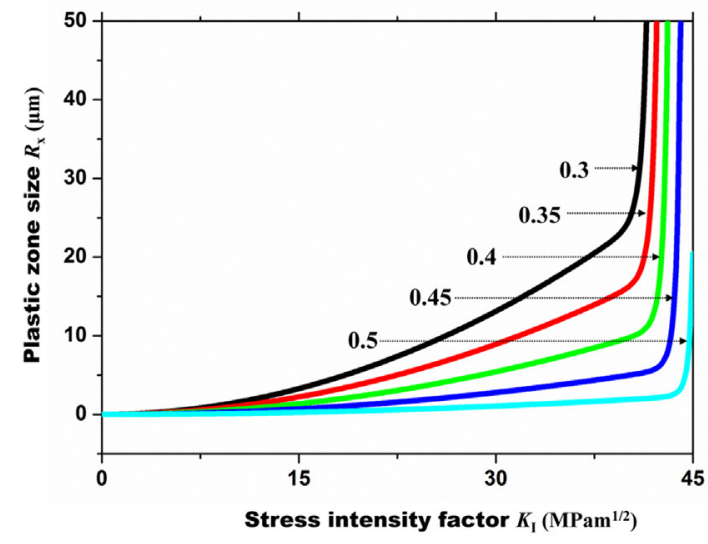

(c)

Fig. 9. Evolutions of the free volume (a), $R_{\max }$ (b), and $R_{x}$ (c) with the stress intensity factor for $\mu=\beta=0.1$ and different values of Poisson's ratio. 


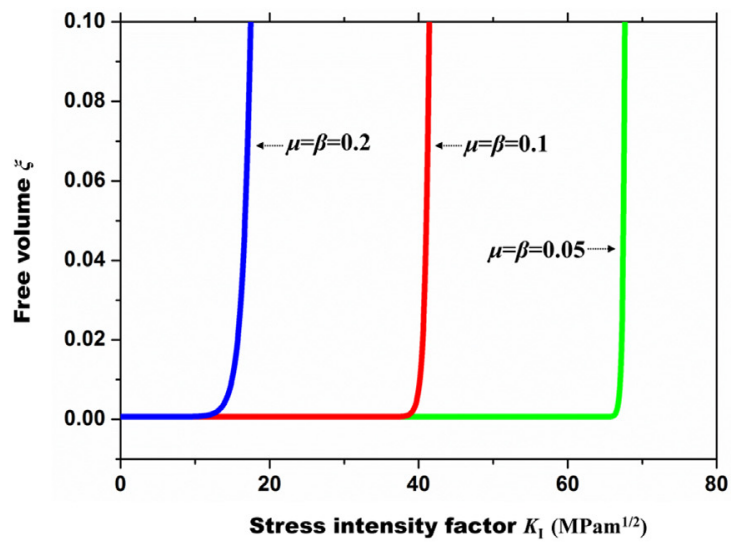

(a)

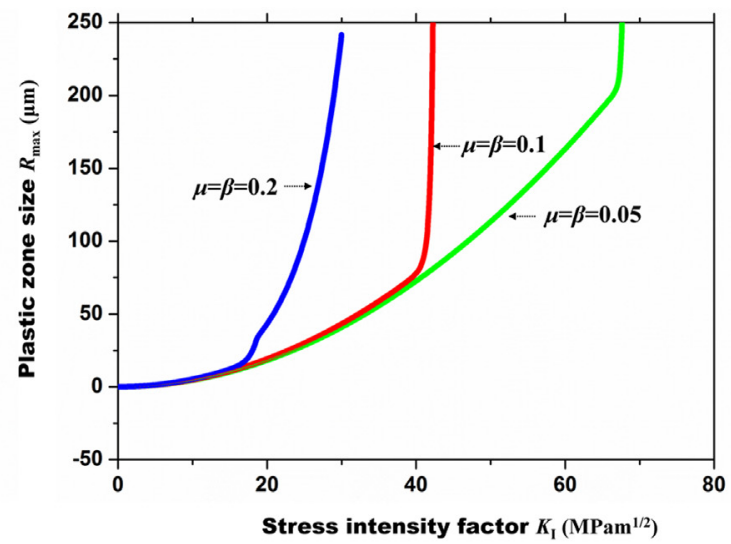

(b)

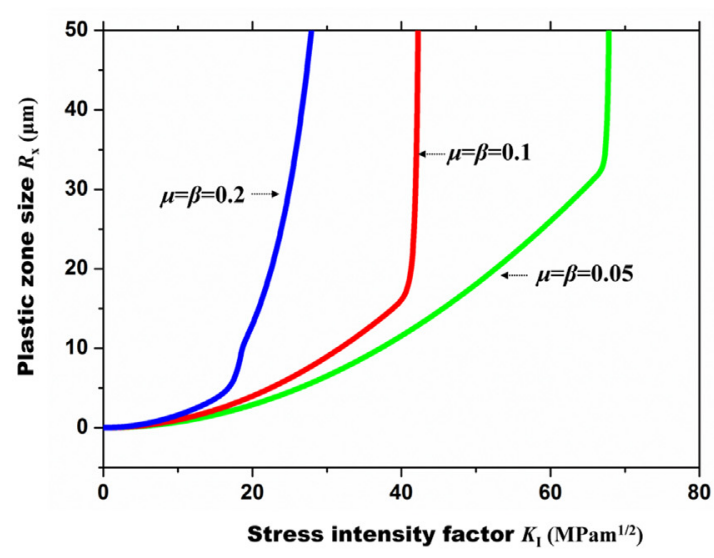

(c)

Fig. 10. Evolutions of the free volume (a), $R_{\max }(\mathrm{b})$, and $R_{x}$ (c) with the stress intensity factor for $v=0.35$ and $\mu=\beta=0.05,0.1$, and 0.2 .

the changes of these material properties have a minor effect on the plastic flow but cause quite a different cavitation behavior in MGs, and finally manipulate the failure mode.

Based on Eqs. (2) and (23), the fracture toughness and the critical plastic zone size for various MGs can be further predicted. Without losing generality, we choose the values of material parameters as the same in Section 3 according to typical MGs such as Zr-based and Cu-based MGs. The stable-to-unstable threshold is determined by the condition that the yield stress reduces to $0.9 \tau_{0}$. The isoline map of the fracture toughness for different shear dilatancy $\beta(\mu=\beta)$ and Poisson's ratio $v$ is illustrated in Fig. 11(a). With the variation of $\beta$ and $v$, the fracture toughness $K_{I c}$ changes from $4 \mathrm{MPam}^{1 / 2}$ to $100 \mathrm{MPam}^{1 / 2} . K_{I c}$ increases very slowly with the growth of $v$, but decreases significantly with the growth of $\beta$. Some typical $\mathrm{Zr}$ - and Cu-based MGs are marked as colored pentagrams and diamonds respectively in the figure. We can see that the Zr-based MGs which display a similar value of Poisson's ratio show very different fracture toughnesses varying from $10 \mathrm{MPam}^{1 / 2}$ to nearly $100 \mathrm{MPam}^{1 / 2}$, this is attributed to their different magnitudes of shear dilatancy or pressure sensitivity. For these Zr- and Cubased MGs which have a fracture toughness as high as around $70 \mathrm{MPam}^{1 / 2}$, their magnitudes of shear dilatancy are quite minor (i.e. smaller than 0.05).

The variations of the characteristic length scales $R_{\operatorname{maxc}}$ and $R_{x c}$ of the critical plastic zone with Poisson's ratio and shear dilatancy are plotted in Fig. 11(b and c) respectively. The isolines of $R_{\operatorname{maxc}}$ changes from $1 \mu \mathrm{m}$ to $400 \mu \mathrm{m}$ with the variation of $v$ and $\beta$. We note that $R_{\operatorname{maxc}}$ mainly relies on the shear dilatancy and it almost keep constant with the change of Poisson's ratio. In contrast, $R_{x c}$ ranges from $500 \mathrm{~nm}$ to $160 \mu \mathrm{m}$, smaller than the corresponding $R_{\operatorname{maxc}}$. With the decrease of $\beta, R_{x c}$ increases. Different from $R_{\max }, R_{x c}$ shows a considerable dependence on Poisson's ratio. With the growth of $v, R_{x c}$ reduces rapidly, especially when $v \rightarrow 0.5$. The values of $R_{\operatorname{maxc}}$ and $R_{x c}$ for the MGs marked in Fig. 11(a) can be predicted in these isoline maps (see Fig. 11(b and c)).

The correlation between the plastic zone and fracture morphology were discussed by Spaepen (1975), Agron (1979) and Xi et al. (2005). Xi et al. (2005) related the plastic zone size to the dimple pattern. As we mentioned, there are two characteristic lengths of the plastic zone. The question raised immediately is that, which one is corresponding to the dimple size? The material properties and dimple sizes for a variety of MGs are collected in Table 1 . The values of $R_{\operatorname{maxc}}$ and $R_{x c}$ are calculated by 


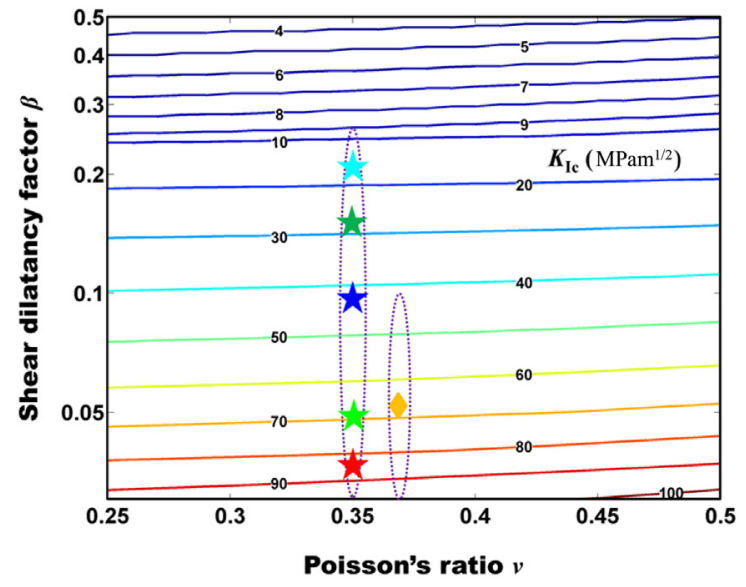

(a)

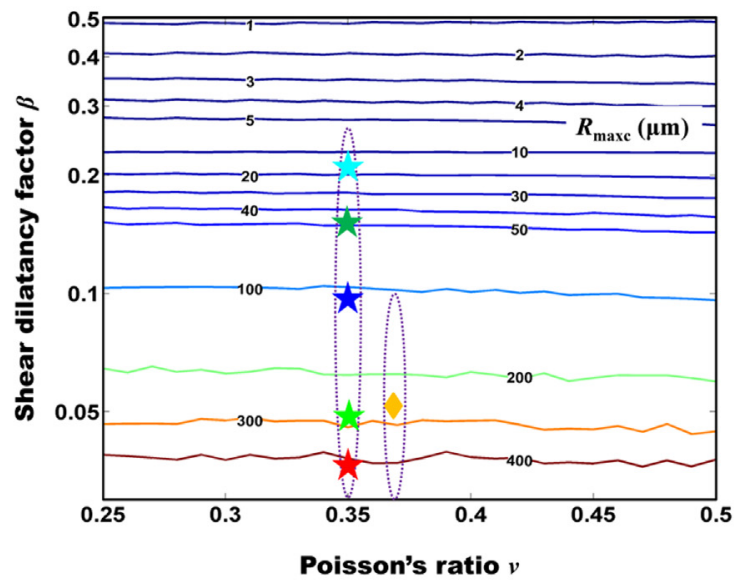

(b)

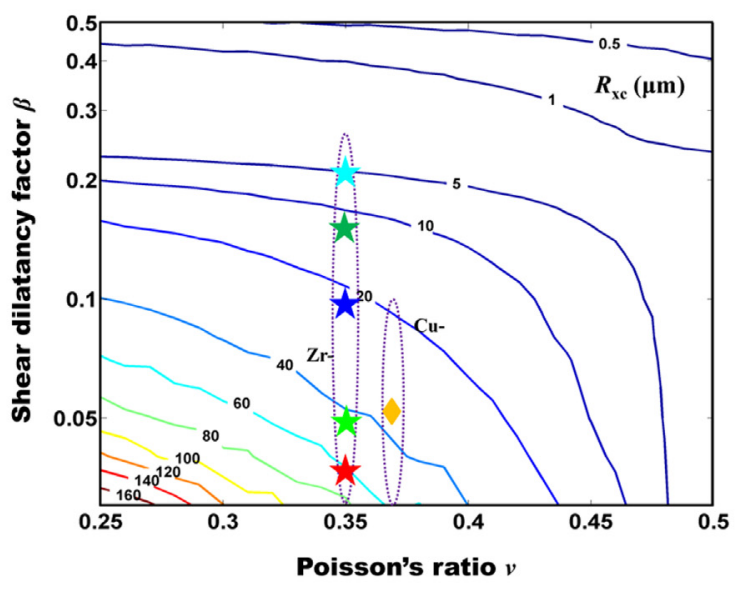

(c)

Fig. 11. Isolines of mode I fracture toughness (a), $R_{\operatorname{maxc}}(\mathrm{b})$, and $R_{x c}$ (c) for different values of shear dilatancy $\beta$ ( $\mu=\beta$ ) and Poission's ratio $v$.

Table 1

Data of $G, \sigma_{y}, \tau_{y}, v, K_{c}, l_{\text {dimple }}$ and the calculated $\beta, R_{\max }, R_{x c}$. A comparison between $R_{x c}$ and $l_{\text {dimple }}$ is given for different MGs. (STZ volume: $\Omega_{\mathrm{Fe}-}=0.5 \times 10^{-27} \mathrm{~m}^{3}$; $\Omega_{\mathrm{Mg}-}=1.0 \times 10^{-27} \mathrm{~m}^{3}$ (Murali et al., 2011a); others $\Omega=1.6 \times 10^{-27} \mathrm{~m}^{3}$ (Li et al., 2013). References: [1] (Conner et al., 1997; Lowhaphandu and Lewandowski, 1998; Lewandowski, 2001; Wang et al., 2002; Lewandowski et al., 2005); [2] (Lewandowski, 2001; Wang et al., 2002); [3] (Jiang et al., 2008a,b); [4] (Wang et al., 2004; Wesseling, 2004; Lewandowski et al., 2005); [5] (Inoue, 2000; Zhang and Inoue, 2001); [6] (Kimura and Masumoto, 1980; Wang et al., 2003; Lewandowski et al., 2005); [7] (Wang et al., 2004; Xi et al., 2005); [8] (Shek et al., 1998).).

\begin{tabular}{|c|c|c|c|c|c|c|c|c|c|c|}
\hline Metallic glasses & $\begin{array}{c}\boldsymbol{G} \\
(\mathrm{GPa})\end{array}$ & $\begin{array}{c}\boldsymbol{\sigma}_{\boldsymbol{y}} \\
(\mathrm{GPa})\end{array}$ & $\begin{array}{c}\boldsymbol{\tau}_{\boldsymbol{y}} \\
(\mathrm{GPa})\end{array}$ & $v$ & $\begin{array}{c}\boldsymbol{K}_{\boldsymbol{c}} \\
\left(\mathrm{MPam}^{1 / 2}\right)\end{array}$ & $\beta$ & $\begin{array}{l}\boldsymbol{R}_{\operatorname{maxc}} \\
(\mu \mathrm{m})\end{array}$ & $\begin{array}{c}\boldsymbol{R}_{\mathbf{x c}} \\
(\mu \mathrm{m})\end{array}$ & $\begin{array}{l}\boldsymbol{l}_{\text {dimple }} \\
(\mu \mathrm{m})\end{array}$ & References \\
\hline$\star \mathrm{Zr}_{41} \mathrm{Ti}_{14} \mathrm{Cu}_{12.5} \mathrm{Ni}_{10} \mathrm{Be}_{22.5}$ & 37.4 & 1.8 & 1.0 & 0.350 & 86 & 0.035 & 411 & 60 & 60 & [1] \\
\hline$\star$ Annealed 0.75h@623K & 37.5 & 1.8 & 1.0 & 0.351 & 68 & 0.05 & 280 & 44 & - & [2] \\
\hline$\star$ Annealed 1.5h @623K & 37.5 & 1.8 & 1.0 & 0.351 & 42.5 & 0.10 & 111 & 23 & - & [2] \\
\hline$\star$ Annealed 3h@623K & 37.5 & 1.8 & 1.0 & 0.347 & 27 & 0.16 & 45 & 12 & - & [2] \\
\hline$\star \mathrm{Zr}_{41.2} \mathrm{Ti}_{13.8} \mathrm{Cu}_{12.5} \mathrm{Ni}_{10} \mathrm{Be}_{22.5}$ & 37.0 & 1.8 & 1.0 & 0.350 & 15 & 0.21 & 16 & 5 & 10 & [3] \\
\hline $\mathrm{Cu}_{60} \mathrm{Zr}_{20} \mathrm{Hf}_{20} \mathrm{Ti}_{10}$ & 36.9 & 1.7 & 0.98 & 0.369 & 67 & 0.05 & 289 & 38 & 35 & [4] \\
\hline $\mathrm{Ti}_{5_{00}} \mathrm{Ni}_{24} \mathrm{Cu}_{20} \mathrm{~B}_{1} \mathrm{Si}_{2} \mathrm{Sn}_{3}$ & 37.0 & 2.1 & 1.2 & 0.350 & 50 & 0.08 & 100 & 19 & 20 & [5] \\
\hline $\mathbf{P d}_{77.5} \mathbf{S i}_{16.5} \mathbf{C u}_{6}$ & 31.9 & 1.57 & 0.91 & 0.410 & 45 & 0.07 & 138 & 13 & 15 & [6] \\
\hline $\mathrm{Ce}_{60} \mathrm{Al}_{20} \mathrm{Ni}_{10} \mathrm{Cu}_{10}$ & 11.5 & 0.65 & 0.38 & 0.313 & 10 & 0.065 & 31 & 7 & 5 & [7] \\
\hline $\mathrm{Mg}_{65} \mathrm{Cu}_{25} \mathrm{~Tb}_{10}$ & 19.6 & 0.66 & 0.38 & 0.309 & 2 & 0.26 & 1.7 & 0.7 & 0.1 & [7] \\
\hline $\mathrm{Fe}_{46} \mathrm{Ni}_{32} \mathrm{~V}_{2} \mathrm{Si}_{14} \mathrm{~B}_{6}$ & 76.1 & 3.8 & 2.2 & 0.314 & 2 & 0.44 & 0.05 & 0.03 & 0.02 & [8] \\
\hline
\end{tabular}




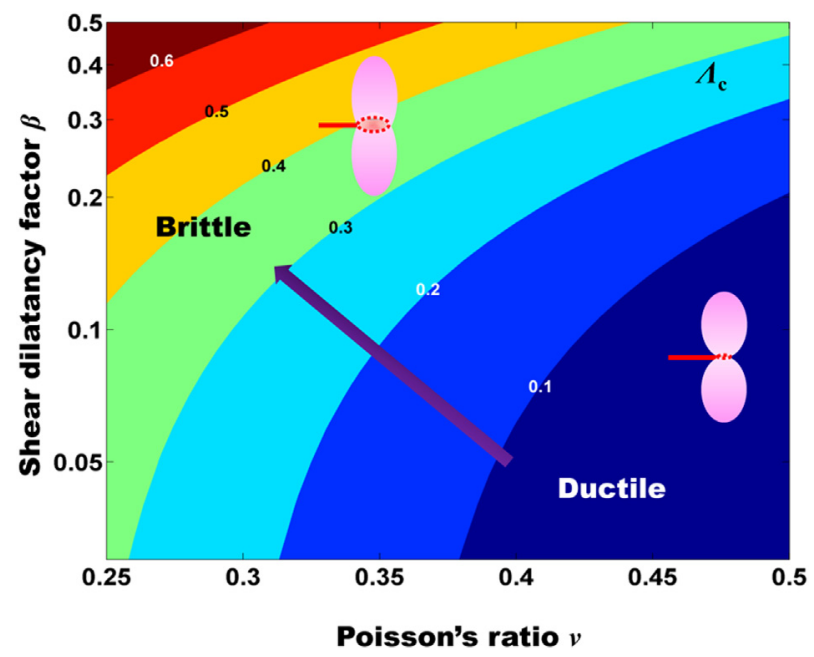

Fig. 12. Isolines of shape factor $\Lambda_{c}$ vary with shear dilatancy $\beta(\mu=\beta)$ and Poisson's ratio $v$.

the present model and they show a wide range for different MGs. It is known that the dimple pattern is usually observed on a mirror-like fracture surface and scales from sub-micrometer to $100 \mathrm{~s}-\mu \mathrm{m}$ (Xi et al., 2005; Yuan et al., 2012). It is a local necking topographic structure and behaves as a typical ductile pattern in brittle fracture. Under mode I load, crack could propagate along the initial direction via cavitation in a brittle mode, the plastic flow within the plastic zone therefore forms a pattern in a scale of $R_{x c}$. It is interesting to find that, for different MGs, the values of $R_{x c}$ and the dimple sizes match each other very well, suggesting that dimple is a flow pattern formed within the plastic zone when a mode I crack opening occurs. In addition, the dilatancy factor $\beta$ is also educed: it ranges from 0.035 to 0.21 for ductile Zr-based MGs, close to these results found by Sun et al. (2010b); as for very brittle Fe-based MGs, the value of $\beta$ can be as high as 0.44 .

The plastic zone shape characterized by $\Lambda_{c}=R_{x c} / R_{\operatorname{maxc}}$ is plotted in Fig. 12, and the isolines for $\Lambda_{c}$ from 0.1 to 0.6 are displayed. We can see that, with the growth of $\beta$ or the decrease of $v, \Lambda_{c}$ increases from 0.1 to 0.6 and plastic zone changes from 'slender' to 'chubby'. For the 'slender' plastic zone (i.e. $R_{x c}$ is far smaller than $R_{\operatorname{maxc}}$ ), the plastic deformation is mainly localized into the fan region and the weak zone ahead the crack tip is very small. It indicates that the plastic zone displays a good flow ability and a restrained area for cavity nucleation and coalescence. In this case, one can expect that the ductile failure caused by shear flow instability should be much easier than the brittle fracture via cavitation. When $R_{x c}$ is approaching to $R_{\operatorname{maxc}}$ or the plastic zone becomes 'chubby', the weak zone for potential cavitation grows and the plastic flow ability becomes relatively poor, promoting the brittle facture. A ductile-to-brittle transition would occur from shear plastic flow to brittle cracking with the increase of $\Lambda_{c}$. The 'slender' plastic zone is found to come along with large Poisson's ratio and small dilatancy factor while these properties are usually shown in ductile MGs (Schroers and Johnson, 2004; Lewandowski et al., 2005; Sun et al., 2010b; Chen et al., 2011). From the map, for Poisson's ratio bigger than 0.32 and dilatancy factor smaller than 0.1 (corresponding to ductile MGs), the value of $\Lambda_{c}$ is within 0.2 (i.e. $R_{x c}$ is smaller than $1 / 5$ of $R_{\operatorname{maxc}}$ ). From the viewpoint of the plastic zone shape, Fig. 12 describes the ductile-to-brittle transition in MGs, and it is also validated by a good consistency of $R_{x c}$ with the dimple size for a variety of MGs. As is known, a quantitative determination of plastic zone from experiments is quite difficult since the plastic zone is extremely localized into micrometer and even nanometer scales. There are some experimental studies on the crack tip field by Flores and Dauskardt (2006), Tandaiya et al. (2009), Xu et al. (2010), He et al. (2012) and etc. However, how to quantitatively characterize the plastic zone ahead of the crack tip is still largely ambiguous, which requires more elaborate experiments with high resolution and effective characterization methods. A further direct validation deserves an experimental study in our later work.

\section{Summary}

In this work, we derived an analytical solution of the plastic zone for mode I crack under plain strain condition through $J$ integral based on a slip line field analysis and shape approximation, where pressure-sensitivity, dilatancy, and free volume evolution are considered. To unveil the nature of plastic zone, two characteristic length scales, i.e. the maximum radius $R_{\text {max }}$ and the radius along the crack line direction $R_{x}$, are proposed and the two lengths are revealed to be involved with the shear flow instability and cavitation, respectively. The influences of Poisson's ratio, pressure sensitivity, and shear dilatancy on the stress/strain distributions and the two lengths are analyzed. It is found that smaller Poisson's ratio and larger pressure sensitivity enable a faster growth of plastic zone, however, this unnecessary leads to a larger plastic zone prior to cracking. The critical values of the mode I stress intensity factor and the plastic zone size at crack initiation are obtained, combined with the free volume evolution from STZ activation. It shows that, smaller dilatancy leads to higher fracture toughness and larger 
critical plastic zone, and increasing Poisson's ratio causes a growth of fracture toughness, but a little change in $R_{\text {maxc }}$ and a decrease in $R_{x c}$. In contrast to $R_{\operatorname{maxc}}$ (relating to shear flow instability), $R_{x c}$ (relating to cavitation) is found to be much more sensitive to the change of Poisson's ratio and dilatancy factor. The values of $R_{x c}$ are consistent with the dimple sizes for different MGs observed from the experiments, indicating that the dimple is a typical pattern formed within the plastic zone when a mode I cracking occurs. What's further, the shape factor is raised by the ratio of $R_{x}$ to $R_{\max }$, which not only depicts the plastic zone shape but also reveals the underlying competition between ductile and brittle failure modes. As for the critical plastic zone at cracking, a ductile-to-brittle transition map relating to the shape factor $\Lambda_{c}$ is derived: $\Lambda_{c}$ increases with the growth of $\beta$ or the decrease of $v$, and the critical plastic zone varies from 'slender' to 'chubby'. It is elucidated that ductile failure via shear plastic flow prefers in 'slender' plastic zone while brittle fracture via cavitation is more likely to occur in 'chubby' plastic zone. The transition of failure modes is revealed to be ascribed to the 'slender'-to-'chubby' change of the critical plastic zone or the competition between the two characteristic lengths, which should provide new insights into the underlying mechanism of ductile-to-brittle transition in MGs.

\section{Acknowledgment}

The work is supported by the Nature Science Foundation of China (Grants Nos. 11572324, 11202221, 11132011 and 11472287), the National Basic Research Program of China (Grants No. 2012CB937500), and the CAS/SAFEA International Partnership Program for Creative Research Teams.

\section{Appendix A. Slip line field analysis}

With the help of the Mohr diagram, the traces of the planes for which Eq. (9) holds form the $\tilde{\alpha}$ lines and the $\tilde{\beta}$ lines in the $\mathrm{x}$ y plane (see Fig. 1(b) and (c)), with one family intersected with the other by an acute angle $\pi / 2-\phi$. The two families of lines incline at angle of $\pi / 4+\phi / 2$ from the major principal axis. We note that $\phi$ is the internal friction angle, the two families of lines are perpendicular to each other when $\phi=0$. Integration of the equilibrium equations along the slip lines shows that

$$
\chi-\varpi=\text { const } \quad \text { (along } \tilde{\alpha} \text { lines), }
$$

and

$$
\chi+\varpi=\text { const } \quad(\text { along } \tilde{\beta} \text { lines) }
$$

where

$$
\chi=-\frac{1}{2 \tan \varphi} \ln \left(1-\frac{\sigma_{m}}{c} \tan \phi\right), \sigma_{m}=\frac{\sigma_{r r}+\sigma_{\theta \theta}}{2},
$$

and $\varpi$ is the angle between the major principal stress axis and the x-axis. In front of the crack tip, the field consists of two constant stress zones (regions I and III) and a centered fan zone (region II), as shown in Fig. 1(c), where an upper part of the model is depicted due to symmetry. Combining the traction-free boundary conditions in region III, we can obtain the complete crack-tip stress fields expressed in the polar coordinates as these reported by Li and Pan (1990a):

$$
\sigma_{i i}=c \cdot \tilde{\sigma}_{i i}(\phi, \theta)
$$

The expressions of $\tilde{\sigma}_{i i}(\phi, \theta)$ respectively for regions I,II, and III are expressed as follows:

In region $\mathrm{I}(0 \leq \theta \leq \pi / 4-\phi / 2)$,

$$
\begin{aligned}
& \tilde{\sigma}_{r r}=\cos \phi \cdot\left(\frac{1}{\sin \phi}-\frac{\exp (-\pi \tan \phi)}{\sin \phi(1+\sin \phi)}-\frac{\exp (-\pi \tan \phi)}{1+\sin \phi} \cos 2 \theta\right) \\
& \tilde{\sigma}_{\theta \theta}=\cos \phi \cdot\left(\frac{1}{\sin \phi}-\frac{\exp (-\pi \tan \phi)}{\sin \phi(1+\sin \phi)}+\frac{\exp (-\pi \tan \phi)}{1+\sin \phi} \cos 2 \theta\right) \\
& \tilde{\tau}_{r \theta}=\cos \phi \frac{\exp (-\pi \tan \phi)}{1+\sin \varphi} \sin 2 \theta
\end{aligned}
$$

In region II $(\pi / 4-\phi / 2 \leq \theta \leq 3 \pi / 4-\phi / 2)$ 


$$
\begin{aligned}
& \tilde{\sigma}_{r r}=\cos \phi \cdot\left(\frac{1}{\sin \phi}-\frac{1+\sin ^{2} \phi}{\sin \phi(1+\sin \phi)} \exp \left(-2 \tan \phi\left(\frac{3}{4} \pi-\theta-\frac{\phi}{2}\right)\right)\right) \\
& \tilde{\sigma}_{\theta \theta}=\cos \phi \cdot\left(\frac{1}{\sin \phi}-\frac{1-\sin \phi}{\sin \phi} \exp \left(-2 \tan \phi\left(\frac{3}{4} \pi-\theta-\frac{\phi}{2}\right)\right)\right) \\
& \tilde{\tau}_{r \theta}=\frac{\cos ^{2} \phi}{1+\sin \phi} \exp \left(-2 \tan \phi\left(\frac{3}{4} \pi-\theta-\frac{\phi}{2}\right)\right) .
\end{aligned}
$$

In region III $(\pi / 4-\phi / 2 \leq \theta \leq \pi)$

$$
\begin{aligned}
& \tilde{\sigma}_{r r}=\frac{\cos \phi}{1+\sin \phi}(1+\cos 2 \theta) \\
& \tilde{\sigma}_{\theta \theta}=\frac{\cos \phi}{1+\sin \phi}(1-\cos 2 \theta) \\
& \tilde{\tau}_{r \theta}=-\frac{\cos \phi}{1+\sin \phi} \sin 2 \theta
\end{aligned}
$$

The closed-form slip line solution is valid for $\phi<\pi / 2$. It is worth to note that these slip line solutions show the similar forms as these of Li and Pan (1990a), however, the important parameters, i.e. the cohesion $c$ and the angle of internal friction $\phi$, have different expressions (see Eqs. (10) and (11)). These solutions obtained from non-normal flow assumption can be reduced into those for normal flow by setting $\beta=\mu$.

\section{Appendix B. Shape approximation of plastic zone}

As for a sharp crack, the near tip field for mode I loading from a linear elastic analysis has the form (Lawn and Wilshaw, 1975):

$$
\sigma_{i j}=\frac{K_{I}}{(2 \pi r)^{1 / 2}} f_{i j}(\theta)+o(r),(i, j=1,2)
$$

with

$$
\left.\begin{array}{l}
f_{11}(\theta) \\
f_{22}(\theta) \\
f_{12}(\theta)
\end{array}\right\}=\cos \frac{\theta}{2}\left\{\begin{array}{l}
1-\sin (\theta / 2) \sin (3 \theta / 2) \\
1+\sin (\theta / 2) \sin (3 \theta / 2) \\
\sin (\theta / 2) \cos (3 \theta / 2)
\end{array}\right\}
$$

For the plane strain state, $\sigma_{33}=v\left(\sigma_{11}+\sigma_{22}\right)$.

According to the yield criterion, when the stress state satisfies Eq. (1), the material starts to experience plastic deformation. Here the effective shear stress and the mean stress are respectively,

$$
\tau_{e}=\sqrt{s_{i j} s_{i j} / 2}=\left[\sin ^{2} \frac{\theta}{2}+\frac{1}{3}(1-2 v)^{2}\right]^{1 / 2} \cdot \frac{K_{I}}{\sqrt{2 \pi r}} \cos \frac{\theta}{2},
$$

and

$$
\sigma_{m}=\sigma_{i i} / 3=\frac{1}{3}(2+2 v) \cdot \frac{K_{I}}{\sqrt{2 \pi r}} \cos \frac{\theta}{2}
$$

Introducing Eqs. (16) and (17) into yield criterion Eq. (1), we have the initial yield radius

$$
r_{y}=\frac{1}{2 \pi}\left(\frac{K_{I}}{\tau_{y}}\right)^{2} g(\theta)
$$

with

$$
g(\theta)=\cos ^{2} \frac{\theta}{2}\left\{\sin ^{2} \frac{\theta}{2}+\frac{1}{3}(1-2 v)^{2}+\frac{4 \mu^{2}}{9}(1+v)^{2}+\frac{4 \mu}{3}(1+v)\left[\sin ^{2} \frac{\theta}{2}+\frac{1}{3}(1-2 v)^{2}\right]^{1 / 2}\right\} .
$$




\section{References}

Anand, L., Su, C., 2005. A theory for amorphous viscoplastic materials undergoing finite deformations, with application to metallic glasses. J. Mech. Phys. Solids 53, 1362-1396.

Argon, A.S., 1979. Plastic deformation in metallic glasses. Acta Metall. 27, 47-58.

Argon, A.S., Salama, M., 1976. The mechanism of fracture in glassy materials capable of some inelastic deformation. Mater. Sci. Eng. 23, 219-230.

Ashby, M., Greer, A., 2006. Metallic glasses as structural materials. Scr. Mater 54, 321-326.

Basu, S., Van der Giessen, E., 2002. A thermo-mechanical study of mode I small-scale yielding crack-tip fields in glassy polymers. Int. J. Plast. 18, 1395-1423.

Biroli, G., 2014. Disordered solids: in search of the perfect glass. Nat. Phys. 10, 555-556.

Chen, K.W., Lin, J.F., 2010. Investigation of the relationship between primary and secondary shear bands induced by indentation in bulk metallic glasses. Int. J. Plast. 26, 1645-1658.

Chen, Y., Jiang, M.Q., Dai, L.H., 2013. Collective evolution dynamics of multiple shear bands in bulk metallic glasses. Inter. J. Plast. 50, 18-36.

Chen, Y., Jiang, M.Q., Wei, Y.J., Dai, L.H., 2011. Failure criterion for metallic glasses. Philos. Mag. 91, 4536-4554.

Conner, R.D., Rosakis, A.J., Johnson, W.L., Owen, D.M., 1997. Fracture toughness determination for a beryllium-bearing bulk metallic glass. Scr. Mater 37, $1373-1378$.

Dai, L.H., 2012. Shear banding in bulk metallic glasses. In: Dodd, B., Bai, Y.L. (Eds.), Adiabatic Shear Localization: Frontiers and Advances, second ed. Elsevier, London, pp. 311-361.

Dai, L.H., Yang, M., Liu, L.F., Bai, Y.L., 2005. Adiabatic shear banding instability in bulk metallic glasses. Appl. Phys. Lett. 87, 141916.

Demetriou, M.D., Launey, M.E., Garrett, G., Schramm, J.P., Hofmann, D.C., Johnson, W.L., Ritchie, R.O., 2011. A damage-tolerant glass. Nat. Mater. $10,123-128$.

Falk, M.L., Langer, J.S., 1998. Dynamics of viscoplastic deformation in amorphous solids. Phys. Rev. E 57, 7192-7205.

Falk, M.L., Langer, J.S., 2011. Deformation and failure of amorphous, solidlike materials. Annu. Rev. Condens. Matter Phys. 2, $353-373$.

Flores, K.M., Dauskardt, R.H., 2001. mean stress effects on flow localization and failure in a bulk metallic glass. Acta Mater 49, $2527-2537$.

Flores, K.M., Dauskardt, R.H., 2006. Mode II fracture behavior of a Zr-based bulk metallic glass. J. Mech. Phys. Solids 54, $2418-2435$.

Fornell, J., Concustell, A., Suriñach, S., Li, W.H., Cuadrado, N., Gebert, A., Baró, M.D., Sort, J., 2009. Yielding and intrinsic plasticity of Ti-Zr-Ni-Cu-Be bulk metallic glass. Int. J. Plast. 25, 1540-1559.

Goodrich, C.P., Liu, A.J., Nagel, S.R., 2014. Solids between the mechanical extreme of ordered and disordered. Nat. Phys. 10, 578-581.

Greer, A.L., Cheng, Y.Q., Ma, E., 2013. Shear bands in metallic glasses. Mater. Sci. Eng. R 74, 71-132.

Grest, G.S., Cohen, M.H., 1981. Liquids, glasses and the glass transition: a free volume approach. Adv. Chem. Phys. 48, 455-525.

He, Q., Shang, J.K., Ma, E., Xu, J., 2012. Crack-resistance curve of a Zr-Ti-Cu-Al bulk metallic glass with extraordinary fracture toughness. Acta Mater 60, 4940-4949.

Henann, D.L., Anand, L., 2009. Fracture of Metallic Glasses at Notches: Effects of Notch-root Radius and the Ratio of the Elastic Shear Modulus to the Bulk Modulus on Toughness, vol. 57, pp. 6057-6074.

Huang, R., Suo, Z., Prevost, J.H., Nix, W.D., 2002. Inhomogeneous deformation in metallic glasses. J. Mech. Phys. Solids 50, $1011-1027$.

Huang, X., Ling, Z., Dai, L.H., 2013. Cavitation instabilities in bulk metallic glasses. Int. J. Solids Struct. 50, 1364-1372.

Huang, X., Ling, Z., Dai, L.H., 2014a. Ductile-to-brittle transition in spallation of metallic glasses. J. Appl. Phys. 116, 143503.

Huang, Y., Khong, J.C., Connolley, T., Mi, J., 2014b. The onset of plasticity of a Zr-based bulk metallic glass. Int. J. Plast. 60, 87-100.

Hutchinson, J.W., 1983. Constitutive behavior and craxk tip fields for materials undergoing creep-constrained grain boundary cavitation. Acta Metall. 31, $1079-1088$.

Inoue, A., 2000. Stabilization of metallic supercooled liquid and bulk amorphous alloys. Acta Mater 48, 279-306.

Jang, D.C., Gross, C.T., Greer, J.R., 2011. Effects of size on the strength and deformation mechanism in Zr-based metallic glasses. Int. J. Plast. $27,858-867$.

Jeong, H.-Y., Li, X.-W., Yee, A.F., Pan, J., 1994. Slip lines in front of a round notch tip in a pressure-sensitive material. Mech. Mater 19, $29-38$.

Jiang, M.Q., Dai, L.H., 2009. On the origin of shear banding instability in metallic glasses. J. Mech. Phys. Solids 57, 1267-1292.

Jiang, M.Q., Dai, L.H., 2011. Shear-band toughness of bulk metallic glasses. Acta Mater 59, 4525-4537.

Jiang, M.Q., Ling, Z., Meng, J.X., Dai, L.H., 2008a. Energy dissipation in fracture of bulk metallic glasses via inherent competition between local softening and quasi-cleavage. Philos. Mag. 88, 407-426.

Jiang, W.H., Fan, G.J., Liu, F.X., Wang, G.Y., Choo, H., Liaw, P.K., 2008b. Spatiotemporally inhomogeneous plastic flow of a bulk-metallic glass. Int. J. Plast. 24, $1-16$.

Johnson, W., Samwer, K., 2005. A universal criterion for plastic yielding of metallic glasses with a $\left(\mathrm{T} / \mathrm{T}_{\mathrm{g}}\right)^{2 / 3}$ temperature dependence. Phys. Rev. Lett. 95, 195501.

Kamrin, K., Bouchbinder, E., 2014. Two-temperature continuum thermomechanics of deforming amorphous solids. J. Mech. Phys. Solids 73, $269-288$.

Kimura, H., Masumoto, T., 1980. Deformation and fracture of an amorphous Pd-Cu-Si Alloy in V-notch bending tests. 2. Ductile-Brittle transition. Acta Metall. 28, 1677-1693.

Lawn, B.R., Wilshaw, T.R., 1975. Fracture of Brittle Solids. Cambridge University Press, Cambridge.

Lewandowski, J.J., 2001. Effects of annealing and changes in stress state on fracture toughness of bulk metallic glass. Mater. Trans. 42, $633-637$.

Lewandowski, J.J., Wang, W.H., Greer, A.L., 2005. Intrinsic plasticity or brittleness of metallic glasses. Philos. Mag. Lett. 85, 77-87.

Li, F.Z., Pan, J., 1990a. Plane-strain crack-tip fields for pressure-sensitive dilatant Materials. J. Appl. Mech. Trans. ASME 57, 40-49.

Li, F.Z., Pan, J., 1990b. Plane-stress crack-tip fields fo pressure-sensitive dilatant materials. Eng. Fract. Mech. 35, 1105-1116.

Li, L., Homer, E.R., Schuh, C.A., 2013. Shear transformation zone dynamics model for metallic glasses incorporating free volume as a state variable. Acta Mater 61, 3347-3359.

Lowhaphandu, P., Lewandowski, J.J., 1998. Fracture toughness and notched toughness of bulk amorphous alloy. Scr. Mater 38, $1811-1817$.

Miracle, D.B., 2004. A structural model for metallic glasses. Nat. Mater 3, 679-702.

Murali, P., Guo, T., Zhang, Y., Narasimhan, R., Li, Y., Gao, H., 2011a. Atomic scale fluctuations govern brittle fracture and cavitation behavior in metallic glasses. Phys. Rev. Lett. 107, 215501.

Murali, P., Guo, T.F., Zhang, Y.W., Narasimhan, R., Li, Y., Gao, H.J., 2011b. Atomic scale fluctuations govern brittle fracture and cavitation behavior in metallic glasses. Phys. Rev. Lett. 107, 215501.

Narasimhan, R., Tandaiya, P., Singh, I., Narayan, R.L., Ramamurty, U., 2015. Fracture in metallic glasses: mechanics and mechanisms. Int. J. Fract. $191,53-75$.

Narayan, R.L., Tandaiya, P., Narasimhan, R., Ramamurty, U., 2014. Wallner lines, crack velocity and mechanisms of crack nucleation and growth in a brittle bulk metallic glass. Acta Mater 80, 407-420.

Nielsen, M.P., 1984. Limit Analysis and Concrete Plasticity. Prentice-Hall, Englewood Cliffs, New Jersey.

Pampillo, C.A., Reimschuessel, A.C., 1974. The Fracture Topography of Metallic Glasses, vol. 9, p. 7.

Pan, J., Shih, C.F., 1986. Plane-strain crack-tip fields for power-law hardening orthotropic materials. Mech. Mater 5, $299-316$.

Pan, J., Shih, C.F., 1988. Plane-stress crack-tip fields for power-law hardening orthotropic materials. Int. J. Fract. 37, 171-195.

Raghavan, R., Murali, P., Ramamurty, U., 2009. On factors influencing the ductile-to-brittle transition in a bulk metallic glass. Acta Mater 57, 3332-3340.

Ravichandran, G., Molinari, A., 2005. Analysis of shear banding in metallic glasses under bending. Acta Mater 53, 4087-4095.

Rice, J.R., 1968. A path-independent integral and the approximate analysis of strain concentration by notches and cracks. J. Appl. Mech. 35, 379-386.

Ritchie, R.O., 2011. The conflicts between strength and toughness. Nat. Mater. 10, 817-822.

Rudnicki, J.W., Rice, J.R., 1975. Conditions for the localization of deformation in pressure-sensitive dilatant materials. J. Mech. Phys. Solids 23, $371-394$.

Rycroft, C., Bouchbinder, E., 2012. Fracture toughness of metallic glasses: annealing-induced embrittlement. Phys. Rev. Lett. 109.

Schroers, J., Johnson, W.L., 2004. Ductile bulk metallic glass. Phys. Rev. Lett. 93, 255506. 
Schuh, C., Hufnagel, T., Ramamurty, U., 2007. Mechanical behavior of amorphous alloys. Acta Mater 55, 4067-4109.

Schuh, C.A., Lund, A.C., 2003. Atomistic basis for the plastic yield criterion of metallic glass. Nat. Mater 2, 449-452.

Shek, C.H., Lin, G.M., Lee, K.L., Lai, J.K.L., 1998. Fractal fracture of amorphous $\mathrm{Fe}_{46} \mathrm{Ni}_{32} \mathrm{~V}_{2} \mathrm{Si}_{14} \mathrm{~B}_{6}$ alloy. J. Non-Cryst. Solids 224, $244-248$.

Singh, I., Guo, T.F., Murali, P., Narasimhan, R., Zhang, Y.W., Gao, H.J., 2013. Cavitation in materials with distributed weak zones: implications on the origin of brittle fracture in metallic glasses. J. Mech. Phys. Solids 61, 1047-1064.

Singh, I., Guo, T.F., Narasimhan, R., Zhang, Y.W., 2014. Cavitation in brittle metallic glasses - effects of stress state and distributed weak zones. Int. J. Solids Struct. 51, 4373-4385.

Spaepen, F., 1975. On the fracture morphology of metallic glasses. Acta Metall. 23, 6.

Spaepen, F., 1977. A microscopic mechanism for steady state inhomogeneous flow in metallic glasses. Acta Metall. 25, 407-415.

Subramanya, H., Viswanath, S., Narasimhan, R., 2007. A three-dimensional numerical study of mode I crack tip fields in pressure sensitive plastic solids. Int. J. Solids Struct. 44, 1863-1879.

Sun, B.A., Yu, H.B., Jiao, W., Bai, H.Y., Zhao, D.Q., Wang, W.H., 2010a. Plasticity of ductile metallic glasses: a self-organized critical state. Phys. Rev. Lett. 105 035501.

Sun, L., Jiang, M.Q., Dai, L.H., 2010b. Intrinsic correlation between dilatation and pressure sensitivity of plastic flow in metallic glasses. Scr. Mater 63, 945-948.

Tandaiya, P., Narasimhan, R., Ramamurty, U., 2007. Mode I crack tip fields in amorphous materials with application to metallic glasses. Acta Mater 55 , $6541-6552$.

Tandaiya, P., Narasimhan, R., Ramamurty, U., 2013. On the mechanism and the length scales involved in the ductile fracture of a bulk metallic glass. Acta Mater 61, 1558-1570.

Tandaiya, P., Ramamurty, U., Narasimhan, R., 2009. Mixed mode (I and II) crack tip fields in bulk metallic glasses. J. Mech. Phys. Solids 57, 1880-1897.

Tandaiya, P., Ramamurty, U., Ravichandran, G., Narasimhan, R., 2008. Effect of Poisson's ratio on crack tip fields and fracture behavior of metallic glasses Acta Mater 56, 6077-6086.

Thamburaja, P., Ekambaram, R., 2007. Coupled thermo-mechanical modelling of bulk-metallic glasses: theory, finite-element simulations and experimental verification. J. Mech. Phys. Solids 55, 1236-1273.

Wang, G., Han, Y.N., Xu, X.H., Ke, F.J., Han, B.S., Wang, W.H., 2008. Ductile to brittle transition in dynamic fracture of brittle bulk metallic glass. J. Appl. Phys. 103.

Wang, G., Wang, Y.T., Liu, Y.H., Pan, M.X., Zhao, D.Q., Wang, W.H., 2006. Evolution of nanoscale morphology on fracture surface of brittle metallic glass. Appl. Phys. Lett. 89, 121909.

Wang, G., Zhao, D.Q., Bai, H.Y., Pan, M.X., Xia, A.L., Han, B.S., Xi, X.K., Wu, Y., Wang, H.W., 2007. Nanoscale periodic morphologies on the fracture surface of brittle metallic glasses. Phys. Rev. Lett. 98, 235501.

Wang, R.J., Li, F.Y., Wang, J.F., Wang, W.H., 2003. Responses of glassy structure and properties to pressure and devitrification. Appl. Phys. Lett. 83, 2814.

Wang, W.H., Dong, C., Shek, C.H., 2004. Bulk metallic glasses. Mater. Sci. Eng. R 44, 45-89.

Wang, W.H., Wang, R.J., Yang, W.T., Wei, B.C., Wen, P., Zhao, D.Q., Pan, M.X., 2002. Stability of ZrTiCuNiBe bulk metallic glass upon isothermal annealing near the glass transition temperature. J. Mater. Res. 17, 1385-1389.

Wesseling, P., 2004. Preliminary assessment of flow, notch toughness, and high temperature behavior of $\mathrm{Cu}_{60} \mathrm{Zr}_{20} \mathrm{Hf}_{10} \mathrm{Ti}_{10}$ bulk metallic glass. Scr. Mater 51 , 151-154.

Wu, F.F., Zheng, W., Wu, S.D., Zhang, Z.F., Shen, J., 2011. Shear stability of metallic glasses. Int. J. Plast. 27, 560-575.

Wu, T.H., 1966. Soil Mechanics. Allyn and Bacon, Boston, Mass.

Wu, Y., Bei, H., Wang, Y.L., Lu, Z.P., George, E.P., Gao, Y.F., 2015. Deformation-induced spatiotemporal fluctuation, evolution and localization of strain fields in a bulk metallic glass. Int. J. Plast. 71, 136-145.

Xi, X.K., Zhao, D.Q., Pan, M.X., Wang, W.H., Wu, Y., Lewandowski, J.J., 2005. Fracture of brittle metallic glasses: brittleness or plasticity. Phys. Rev. Lett. 94, 125510.

Xi, X.K., Zhao, D.Q., Pan, M.X., Wang, W.H., Wu, Y., Lewandowski, J.J., 2006. Periodic corrugation on dynamic fracture surface in brittle bulk metallic glass. Appl. Phys. Lett. 89, 181911.

Xia, X.X., Wang, W.H., 2012. Characterization and modeling of breaking-induced spontaneous nanoscale periodic stripes in metallic glasses. Small 8 $1197-1203$

Xu, J., Ma, E., 2014. Damage-tolerant Zr-Cu-Al-based bulk metallic glasses with record-breaking fracture toughness. J. Mater. Res. 29, 1489-1499.

Xu, J., Ramamurty, U., Ma, E., 2010. The fracture toughness of bulk metallic glasses. Jom-US 62, 10-18.

Yang, Q., Mota, A., Ortiz, M., 2006. A finite-deformation constitutive model of bulk metallic glass plasticity. Comput. Mech. 37, 194-204.

Yuan, C.C., Ma, J., Xi, X.K., 2012. Understanding the correlation of plastic zone size with characteristic dimple pattern length scale on the fracture surface of a bulk metallic glass. Mater. Sci. Eng. A 532, 430-434.

Zhang, T., Inoue, A., 2001. Ti-based amorphous alloys with a large supercooled liquid region. Mater. Sci. Eng. A 304-306, 771-774.

Zhao, P., Li, J., Wang, Y., 2013. Heterogeneously randomized STZ model of metallic glasses: softening and extreme value statistics during deformation. Int. J. Plast. 40, 1-22. 